\title{
ON THE NONEXISTENCE OF PURE MULTI-SOLITONS FOR THE QUARTIC GKDV EQUATION
}

\author{
YVAN MARTEL AND FRANK MERLE
}

Abstract. We consider the quartic (nonintegrable) (gKdV) equation

$$
\partial_{t} u+\partial_{x}\left(\partial_{x}^{2} u+u^{4}\right)=0, \quad t, x \in \mathbb{R},
$$

and $u(t)$ an outgoing 2 -soliton of the equation, i.e. a solution satisfying

$$
\lim _{t \rightarrow+\infty}\left\|u(t)-Q_{c_{1}}\left(.-c_{1} t\right)-Q_{c_{2}}\left(.-c_{2} t\right)\right\|_{H^{1}}=0,
$$

where $0<c_{2}<c_{1}$ and where $Q_{c_{j}}\left(x-c_{j} t\right)$ are explicit solitons of the equation.

In [19], in the case $0<1-\frac{c_{2}}{c_{1}}=\epsilon \leq \epsilon_{0}$, where $\epsilon_{0}$ is a small enough, not explicit constant, the solution $u(t, x)$ is computed up to some order of $\epsilon$, for all $t$ and $x$. In particular, it is deduced that $u(t)$ is not a multi-soliton as $t \rightarrow-\infty$, proving the nonexistence of pure multi-soliton in this context.

In the present paper, we prove the same result for an explicit range of speeds: $\frac{3}{4} c_{1}<c_{2}<c_{1}$, by a different approach, which does not longer require a precise description of the solution. In fact, the nonexistence result holds for outgoing $N$-solitons, for any $N \geq 2$, under the assumption: $\sum_{j=2}^{N}\left(1-c_{j} / c_{1}\right)^{2} \leq \frac{1}{16}$ which is a natural generalization of the condition for $N=2$.

\section{Introduction}

1.1. Setting of the problem. In this paper, we focus on the quartic generalized Korteweg-de Vries (gKdV) equation

$$
\partial_{t} u+\partial_{x}\left(\partial_{x}^{2} u+u^{4}\right)=0, \quad t, x \in \mathbb{R} .
$$

Recall that the Cauchy problem for (1.1) is globally well-posed in $H^{1}$ (see Kenig, Ponce and Vega [6] for a precise existence and uniqueness statement), and that any $H^{1}$ solution $u(t, x)$ of (1.1) satisfies for all $t \in \mathbb{R}$,

$$
\begin{aligned}
& \int u^{2}(t)=M(u(t))=M(u(0)) \quad(\text { mass }) \\
& \int\left(\partial_{x} u\right)^{2}(t)-\frac{2}{5} u^{5}(t)=E(u(t))=E(u(0)) \quad(\text { energy })
\end{aligned}
$$

Recall also that the integral of $u(t)$ is preserved provided it is well-defined:

$$
\int u(t)=\int u(0)
$$

We call soliton a solution of (1.1) of the form

$$
R_{c, y_{0}}(t, x)=Q_{c}\left(x-c t-y_{0}\right), \quad \text { for } c>0, y_{0} \in \mathbb{R},
$$


where $Q_{c}(x)=c^{\frac{1}{3}} Q(\sqrt{c} x)$ and $Q$ satisfies

$$
Q^{\prime \prime}+Q^{4}=Q, \quad Q(x)=\left(\frac{5}{2 \cosh ^{2}\left(\frac{3}{2} x\right)}\right)^{\frac{1}{3}} .
$$

We call outgoing multi-soliton a solution $u(t)$ of (1.1) such that

$$
\lim _{t \rightarrow+\infty}\left\|u(t)-\sum_{j=1}^{N} Q_{c_{j}}\left(.-c_{j} t-\Delta_{j}\right)\right\|_{H^{1}}=0
$$

for some $N \geq 2,0<c_{N}<\ldots<c_{1}$, and $\Delta_{j} \in \mathbb{R}$. For a given set of such parameters, the existence and uniqueness of an outgoing multi-soliton was proved in [1] (see also [21] for previous related results), together with the following regularity and convergence properties: $u(t) \in \cap_{s \geq 1} H^{s}$, and for some $\gamma>0$, for all $s \geq 0$,

$$
\text { for all } t>0, \quad\left\|u(t)-\sum_{j=1}^{N} Q_{c_{j}}\left(.-c_{j} t-\Delta_{j}\right)\right\|_{H^{s}} \leq C_{s} e^{-\gamma t} \text {. }
$$

Similarly, we call ingoing multi-soliton, a solution $u(t)$ of (1.1) such that

$$
\lim _{t \rightarrow-\infty}\left\|u(t)-\sum_{j=1}^{N} Q_{c_{j}}\left(.-c_{j} t-\Delta_{j}\right)\right\|_{H^{1}}=0 .
$$

We call pure multi-soliton, a solution of (1.1) which is both an ingoing and an outgoing multi-soliton, possibly with different numbers of solitons $N^{ \pm}$and different speeds and position parameters $c_{j}^{ \pm}, \Delta_{j}^{ \pm}$as $t \rightarrow+\infty$ or as $t \rightarrow-\infty$. The aim of this paper is to investigate the relation between ingoing and outgoing multi-solitons of the nonintegrable quartic ( $\mathrm{gKdV}$ ) equation (1.1), and more precisely to prove the nonexistence of pure multi-solitons for an explicit range of speeds.

It is well-known that for the $(\mathrm{KdV})$ and $(\mathrm{mKdV})$ equations, i.e. in the integrable cases,

$$
\begin{array}{lll}
\partial_{t} u+\partial_{x}\left(\partial_{x}^{2} u+u^{2}\right)=0 & & (\mathrm{KdV}) \\
\partial_{t} u+\partial_{x}\left(\partial_{x}^{2} u+u^{3}\right)=0 & & (\mathrm{mKdV})
\end{array}
$$

this question was completely settled by integrability (see e.g. [2, 35, 36, 9, 4, 32, 22]). Indeed, there exist explicit pure multi-solitons for any parameters and they are the only ingoing multi-solitons. In particular, the collision of any number of solitons is always elastic, meaning that neither the number of solitons, nor their speeds, are changed by the collision (the trajectories of the solitons are in general shifted). We refer to [22] and references therein for a review of results for the integrable models (1.7), (1.8).

For nonintegrable models, existence and properties of multi-solitons has also become a classical question, studied through different points of view (see e.g. [22, 29, 21, 23, 1, 3]). For the quartic $(\mathrm{gKdV})$ equation, the authors of the present paper have already adressed this question in the case of 2 -solitons with speeds $0<c_{2}<c_{1}$ in the following two cases, for $\epsilon>0$ small:

(a) Solitons of different speeds: $\frac{c_{2}}{c_{1}} \leq \epsilon$. See [18]

(b) Solitons with almost equal speeds: $0<1-\frac{c_{2}}{c_{1}} \leq \epsilon$. See [19]. 
In [18] and [19], under condition (a) or (b), we have given a refined description of ingoing 2-solitons for all $t$ and $x$, up to some order of $\epsilon$. From this description, we could deduce the following facts. (1) The 2-soliton structure is globally stable in time in $H^{1}$, in the sense that an ingoing 2-soliton is for all time the sum of two solitons at the main order. (2) Ingoing 2-solitons cannot be outgoing 2-solitons. In particular, no pure 2-soliton can exist in these two regimes. In contrast with the integrable cases, the collision is inelastic. From explicit computations, we could find lower bounds and upper bounds on the size of the residual term due to the collision.

Summarizing, ingoing 2-solitons are well-understood for all time under assumptions (a) and (b) for $\epsilon>0$ small enough. However, the value of $\epsilon$ for which the results in [18 and [19] hold is not explicit because of the complexity of the computations and the perturbative nature of the proofs. Another restriction concerns the number of solitons. In [18] and in [19], the proofs are only written for 2-solitons and it would be quite involved to extend them to $N$-solitons.

In view of the inelasticity results in [18] and [19], we conjecture that for all $0<c_{2}<c_{1}$, the corresponding ingoing 2 -solitons of the quartic ( $\left.\mathrm{gKdV}\right)$ equation are not pure 2-solitons. In other words, there should not exist pure 2-solitons. In fact, we expect that such property is true for general nonintegrable systems. Perelman's work [28] for the nonlinear Schrödinger equation, Munoz' works [24, 25, 26], and [20] for the BBM equation, are other evidences of such belief.

In this paper, we attack the problem through a different strategy. The main point is to prove nonexistence of pure 2-soliton of the quartic ( $\mathrm{gKdV}$ ) equation without trying to describe the solution for all time and for an explicit range of speeds. Here, the approach is not perturbative, and we do not need to compute the main order of the solution for all $t, x$. Indeed, a contradiction is obtained by estimating only the tail of the solution $u(t, x)$ for large $t$ and large $x$. The knowledge of the solution on compact sets of space-time is not required. Moreover, the method allows to consider the case of $N$-solitons, for any $N \geq 2$, without significant changes, in contrast with [18] and [19]. Note that we consider the quartic (gKdV) equation because it is a typical nonintegrable system, relatively simple and not perturbative of the integrable cases (see [30], [7]), but we expect our approach to be general and flexible enough to extend to other models.

1.2. Statement of the result. The following is the main result of this paper.

Theorem 1.1. Let $N \geq 2$. Let $u(t)$ be an outgoing multi-soliton of (1.1), with parameters $\Delta_{1}, \ldots, \Delta_{N} \in \mathbb{R}, 0<c_{N}<\ldots<c_{1}=1$,

$$
\lim _{t \rightarrow+\infty}\left\|u(t)-\sum_{j=1}^{N} Q_{c_{j}}\left(.-c_{j} t-\Delta_{j}\right)\right\|_{H^{1}}=0 .
$$

Assume that

$$
\sum_{j=2}^{N}\left(1-c_{j}\right)^{2}<\frac{1}{16}
$$

Then, $u(t)$ is not an ingoing multi-soliton at $-\infty$.

In particular, under assumption (1.10), there exists no pure multi-soliton of (1.1) with speeds $1, c_{2}, \ldots, c_{N}$ at $+\infty$ or at $-\infty$. 
Remark 1.2. In the case $N=2$, Theorem 1.1 proves the nonexistence of pure 2-soliton under the condition $\frac{3}{4}<\frac{c_{2}}{c_{1}}<1$.

The strategy of the proof is different from the one in [18] and [19] where the goal was to describe ingoing 2 -solitons for all $t, x \in \mathbb{R}$, by a pertubative analysis. In this paper, to prove nonexistence of multi-solitons, we do not need to understand the solution on bounded sets of $(t, x)$ and we only consider the tail of the solution as $|x| \sim+\infty$. This approach involves different computations which we can perfom for an explicit range of speeds and for any number of solitons.

For the sake of contradiction, we assume the existence of a solution $u(t)$ of (1.1) which is both an outgoing multisoliton $(t \rightarrow+\infty)$ with parameters $N \geq 2,0<$ $c_{N}<\ldots<c_{1}, \Delta_{1}, \ldots, \Delta_{N} \in \mathbb{R}:$

$$
\lim _{t \rightarrow+\infty}\left\|u(t)-\sum_{j=1}^{N} Q_{c_{j}}\left(.-c_{j} t-\Delta_{j}\right)\right\|_{H^{1}}=0,
$$

and an ingoing multisoliton $(t \rightarrow-\infty)$ with parameters $N^{-} \geq 2,0<c_{N^{-}}^{-}<\ldots<$ $c_{1}^{-}, \Delta_{1}^{-}, \ldots, \Delta_{N^{-}}^{-} \in \mathbb{R}:$

$$
\lim _{t \rightarrow-\infty}\left\|u(t)-\sum_{j=1}^{N^{-}} Q_{c_{j}^{-}}\left(.-c_{j}^{-} t-\Delta_{j}^{-}\right)\right\|_{H^{1}}=0 .
$$

We assume

$$
c_{1}=1
$$

and

$$
\sum_{j=2}^{N}\left(1-c_{j}\right)^{2} \leq \frac{1}{16}
$$

The contradiction comes from the following steps:

(a) Control of the speeds at $-\infty$. From the three conservation laws (mass, energy and integral) and elementary algebraic arguments, we claim that the speeds at $-\infty$ are also close to 1 in the following sense (see Section 2)

Lemma 1.3. Assume (1.10), (1.11), (1.12). For all $j=1, \ldots, N^{-}$,

$$
\frac{16}{25}<c_{j}^{-}<\frac{3}{2}
$$

Moreover,

$$
\left|N^{-}-N\right| \leq \frac{\sqrt{N}}{8}
$$

(b) Decay on the right for positive time. From the behavior of the solution $u(t)$ as $t \rightarrow-\infty$ in the energy space (1.12), the lower bound $c_{N}^{-}>\frac{16}{25}$ and usual monotonicity arguments, we claim that the solution $u(t)$ satisfies exponential decay property on the right of the soliton $Q\left(x-t-\Delta_{1}\right)$.

Let $j_{0} \in\{1, \ldots, N-1\}$ be such that

$$
\sigma_{0}:=\min _{j} \sqrt{c_{j+1}}\left(c_{j}-c_{j+1}\right)=\sqrt{c_{j_{0}+1}}\left(c_{j_{0}}-c_{j_{0}+1}\right),
$$


and set

$$
\gamma_{0}=\sqrt{c_{j_{0}}-\frac{3}{4} c_{j_{0}+1}}-\frac{1}{2} \sqrt{c_{j_{0}+1}}, \quad x_{0}(t)=\left(\frac{\sigma_{0}}{\gamma_{0}}+c_{j_{0}}\right) t-K_{0},
$$

where $K_{0}>1$ is a large constant to be fixed later. Note by (3.6) that $\frac{\sigma_{0}}{\gamma_{0}}+c_{j_{0}}>$ $2 c_{j_{0}+1}>\frac{3}{2}$. We claim (see Section 3)

Lemma 1.4. There exists $t_{0}\left(K_{0}\right)>0$ such that,

$$
\forall t>t_{0}\left(K_{0}\right), \quad\left|u\left(t, x_{0}(t)\right)\right| \leq e^{-2 \sigma_{0} t} .
$$

(c) Approximate solution and lower bound. We establish the following result, which is the main new ingredient of the paper.

Proposition 1.5. Assume (1.14). There exist $C_{1}>0$ independent of $K_{0}$ and $t_{1}\left(K_{0}\right)>0$ such that, for $K_{0}$ large enough,

$$
\forall t \geq t_{1}\left(K_{0}\right), \quad\left|u\left(t, x_{0}(t)\right)\right| \geq C_{1} e^{\gamma_{0} K_{0}} e^{-2 \sigma_{0} t} .
$$

Fix $K_{0}>0$ such that $C_{1} e^{\gamma_{0} K_{0}}>2$. Combining Lemma 1.4 and Proposition 1.5, we obtain a contradiction for $t>\max \left(t_{0}\left(K_{0}\right), t_{1}\left(K_{0}\right)\right)$.

Let us sketch the proof of Proposition 1.5. The key point is to construct an explicit approximate solution $V(t)$ of the problem as $t \rightarrow+\infty$ (see Section 44). We briefly sketch the construction of $V$ in the 2-soliton case, i.e. for $N=2$. Let

$$
V=R_{1}+R_{2}+Z, \quad R_{1}(t, x)=Q\left(x-t-\Delta_{1}\right), R_{2}(t, x)=Q_{c_{2}}\left(x-c_{2} t-\Delta_{2}\right),
$$

where

$$
Z_{t}+\left(Z_{x x}+4 R_{1}^{3} Z\right)_{x} \approx-4\left(R_{1}^{3} R_{2}\right)_{x}
$$

In the equation of $Z$ above, we focus on the main interaction term (see Section 4 for the control of all error terms). For this term, we replace $R_{2}$ by its asymptotics for $x-c_{2} t \gg 1$ :

$$
R_{1}^{3} R_{2} \approx 10^{\frac{1}{3}} c_{2}^{\frac{1}{3}} e^{-\sqrt{c_{2}}\left(x-c_{2} t-\Delta_{2}\right)} R_{1}^{3}=c_{0} e^{-\sqrt{c_{2}}\left(1-c_{2}\right) t} e^{-\sqrt{c_{2}}\left(x-t-\Delta_{1}\right)} R_{1}^{3},
$$

where $c_{0}=10^{\frac{1}{3}} c_{2}^{\frac{1}{3}} e^{-\sqrt{c_{2}}\left(\Delta_{1}-\Delta_{2}\right)}$. An explicit solution of

$$
Z_{t}+\left(Z_{x x}+4 R_{1}^{3} Z\right)_{x}=-4 c_{0}\left(e^{-\sqrt{c_{2}}\left(1-c_{2}\right) t} e^{-\sqrt{c_{2}}\left(x-t-\Delta_{1}\right)} R_{1}^{3}\right)_{x}
$$

is $Z(t, x)=4 c_{0} e^{-\sqrt{c_{2}}\left(1-c_{2}\right) t} A\left(x-t-\Delta_{1}\right)$ where $A(x)$ satisfies the following ODE

$$
\left(-A^{\prime \prime}+A-4 Q^{3} A\right)^{\prime}+\sqrt{c_{2}}\left(1-c_{2}\right) A=\left(e^{-\sqrt{c_{2}} x} Q^{3}\right)^{\prime} .
$$

Moreover, for $\frac{3}{4}<c_{2}<1$, we prove the following asymptotic property

$$
A(x) \underset{x \rightarrow+\infty}{\sim} a e^{-\gamma_{0} x} \quad \text { where } a \neq 0 \text { and } \gamma_{0}=\sqrt{1-\frac{3}{4} c_{2}}-\frac{1}{2} \sqrt{c_{2}} .
$$

The proof of property (1.22) requires more than standard ODE techniques, and involves Virial type arguments, introduced in [13], [12] and [16] to study the flow of the evolution problem (1.1) near solitons.

It follows from (1.22) that $V$ satisfies the following lower bound, for $\kappa>0, t$ and $x$ large enough,

$$
|V(t, x)| \geq \kappa e^{-\gamma_{0}(x-t)} e^{-\sqrt{c_{2}}\left(1-c_{2}\right) t} .
$$


Such an approximate solution $V(t)$ being constructed (the actual approximate solution is more refined), by usual techniques ([21], [11]), we compare the solution $u(t)$ with $V(t)$, for $t$ large:

$$
\|u(t)-V(t)\|_{H^{1}} \leq C e^{-2 \sqrt{c_{2}}\left(1-c_{2}\right) t}
$$

and we obtain the desired lower bound on $u(t, x)$ at $x=x_{0}(t)$ (see Section 5).

Comment on assumption (1.10). The assumption on the speeds $c_{1}, \ldots, c_{N}$ in (1.10) is not optimal, and we even conjecture that the result holds for any choice of speeds. However, we believe that to obtain the more general result will require much harder analyis. Even considering the simplest case of a 2-soliton with speeds $c_{1}=1$ and $c_{2}$, we see several difficulties to extend the nonexistence result to any $0<c_{2}<1$.

1) For $0<c_{2} \leq 1 / 3$, the method outlined above does not work direclty for algebraic reasons. Indeed, $0<c_{2} \leq 1 / 3$ implies $\gamma_{0} \geq \sqrt{c_{2}}$, and thus the approximate solution has the same decay as $R_{2}$, and no direct contradiction can follow from such a lower bound. This is related to the fact that the proof of inelasticity for $c_{2}$ close to 0 in [18] requires a higher order expansion than the one in [19] for $c_{2}$ close to 1 .

2) For all $\frac{1}{3}<c_{2}<1$, we expect that the function $A(x)$ defined above has the generic decay (1.22), which is essential in our proof, but we were able to prove this fact only for $c_{2} \in\left[c_{0}, 1\right]$, where $c_{0}<\frac{3}{4}$ is close to $\frac{3}{4}$.

3) The restriction (1.10) on the values of $c_{2}$ also comes from the proof of the decay property obtained in Lemma 1.4. We prove Lemma 1.4 by known and simple energy localization arguments, which are clearly not optimal. Replacing these arguments by a sharper asymptotic analysis would certainly improve the range of admissible $c_{2}$, but without approching the special value $c_{2}=\frac{1}{3}$.

In the case of 2-solitons, it is proved in Proposition 2.2 that $N^{-}=2$ and $c_{1}^{-}=1$, $c_{2}^{-}=c_{2}$ without condition on $c_{2}$. For $N \geq 3$, it is not clear how to prove such a rigidity property, or even how to obtain a lower bound such as (1.15) without a strong assumption on the speeds at $+\infty$ such as (1.10). In particular, we cannot replace (1.10) by $\frac{3}{4}<c_{j}<1, \forall j=2, \ldots, N$.

Acknowledgement. This work is partly supported by the project ERC 291214 BLOWDISOL.

\section{Rigidity of multi-soliton parameters}

In this section, using the three conservation laws (1.2), (1.3) and (1.4), we prove Lemma 1.3. which controls the speeds at $-\infty$ for an outgoing multi-soliton under assumption (1.10). We also state and prove an independent unconditional result of rigidity of the speeds at $\pm \infty$ for a 2 -soliton.

Note that the arguments can be extended to other power nonlinearities.

2.1. Conservation laws on ingoing $N$-solitons. We first claim the following result to be proved in Appendix $\mathrm{A}$. 
Lemma 2.1. Let $N \geq 2,0<c_{N}<\ldots<c_{1}$ and $\Delta_{1}, \ldots, \Delta_{N} \in \mathbb{R}$. Let $u(t)$ be the solution of (1.1) satisfying

$$
\lim _{t \rightarrow+\infty}\left\|u(t)-\sum_{j=1}^{N} Q_{c_{j}}\left(.-c_{j} t-\Delta_{j}\right)\right\|_{H^{1}}=0 .
$$

Then, for all $t$,

$$
\begin{aligned}
& \int u^{2}(t)=\sum_{j=1}^{N} \int Q_{c_{j}}^{2}=\left(\sum_{j=1}^{N} c_{j}^{\frac{1}{6}}\right) \int Q^{2} . \\
& E(u(t))=\sum_{j=1}^{N} E\left(Q_{c_{j}}\right)=\left(\sum_{j=1}^{N} c_{j}^{\frac{7}{6}}\right) E(Q) .
\end{aligned}
$$

Moreover, $u(t) \in L^{1}$ and

$$
\int u(t)=\sum_{j=1}^{N} \int Q_{c_{j}}=\left(\sum_{j=1}^{N} c_{j}^{-\frac{1}{6}}\right) \int Q .
$$

2.2. Proof of Lemma 1.3, Using Lemma 2.1 and (1.11)-(1.12), the following identities hold

$$
\sum_{j=1}^{N} c_{j}^{\frac{7}{6}}=\sum_{j=1}^{N^{-}}\left(c_{j}^{-}\right)^{\frac{7}{6}}, \quad \sum_{j=1}^{N} c_{j}^{-\frac{1}{6}}=\sum_{j=1}^{N^{-}}\left(c_{j}^{-}\right)^{-\frac{1}{6}}, \quad \sum_{j=1}^{N} c_{j}^{\frac{1}{6}}=\sum_{j=1}^{N^{-}}\left(c_{j}^{-}\right)^{\frac{1}{6}} .
$$

Consider the function $f(x)$ for $x>0$ defined as

$$
f(x)=x^{7}+\frac{3}{x}-4 x \quad \text { so that } \quad f^{\prime}(x)=7 x^{6}-\frac{3}{x^{2}}-4, \quad f^{\prime \prime}(x)=42 x^{5}+\frac{6}{x^{3}}>0 .
$$

In particular, $f(1)=f^{\prime}(1)=0$ and elementary computations show that

$$
\begin{gathered}
\forall x>0, \quad f^{\prime \prime}(x) \geq f^{\prime \prime}\left((3 / 35)^{\frac{1}{8}}\right)=\frac{48}{5}\left(\frac{35}{3}\right)^{\frac{3}{8}}:=2 m_{1}, \\
\frac{3}{4} \leq x \leq 1 \Rightarrow f^{\prime \prime}(x) \leq f^{\prime \prime}(1)=48 .
\end{gathered}
$$

We deduce:

$$
\forall x>0, f(x) \geq m_{1}(1-x)^{2} ; \quad \forall x \in\left[\frac{3}{4}, 1\right], f(x) \leq 24(1-x)^{2} .
$$

Combining the identities in (2.2), we have

$$
\sum_{j=1}^{N^{-}} f\left(\left(c_{j}^{-}\right)^{\frac{1}{6}}\right)=\sum_{j=1}^{N} f\left(c_{j}^{\frac{1}{6}}\right)
$$


Using (2.3), (1.10) and $1-c_{j}^{\frac{1}{6}} \leq 4\left(1-\left(\frac{3}{4}\right)^{\frac{1}{6}}\right)\left(1-c_{j}\right)\left(\right.$ since $\left.\frac{3}{4}<c_{j}<1\right)$,

$$
\begin{aligned}
& \left|1-\left(c_{j}^{-}\right)^{\frac{1}{6}}\right|^{2} \leq \sum_{j=1}^{N^{-}}\left|1-\left(c_{j}^{-}\right)^{\frac{1}{6}}\right|^{2} \leq \frac{24}{m_{1}} \sum_{j=2}^{N}\left|1-c_{j}^{\frac{1}{6}}\right|^{2} \\
& \leq 80\left(\frac{3}{35}\right)^{\frac{3}{8}}\left(1-\left(\frac{3}{4}\right)^{\frac{1}{6}}\right)^{2} \sum_{j=2}^{N}\left(1-c_{j}\right)^{2} \leq 5\left(\frac{3}{35}\right)^{\frac{3}{8}}\left(1-\left(\frac{3}{4}\right)^{\frac{1}{6}}\right)^{2}:=m_{2} .
\end{aligned}
$$

Thus, for all $j=1, \ldots, N^{-}$, by elementary computations,

$$
\frac{16}{25}<\left(1-\sqrt{m_{2}}\right)^{6} \leq c_{j}^{-} \leq\left(1+\sqrt{m_{2}}\right)^{6}<\frac{3}{2} .
$$

Now, we prove the control of $N^{-}$. Indeed, we have

$$
\begin{aligned}
& \left|\sqrt{N}-\left(\sum_{j=1}^{N} c_{j}^{\frac{1}{6}}\right)^{\frac{1}{2}}=\right|\left(\sum_{j=2}^{N} 1^{2}\right)^{\frac{1}{2}}-\left(\sum_{j=1}^{N}\left|c_{j}^{\frac{1}{12}}\right|^{2}\right)^{\frac{1}{2}} \mid \leq\left(\sum_{j=2}^{N}\left|1-c_{j}^{\frac{1}{12}}\right|^{2}\right)^{\frac{1}{2}} \\
& \leq\left(1+\left(\frac{3}{4}\right)^{\frac{1}{12}}\right)^{-1}\left(\sum_{j=1}^{N}\left|1-c_{j}^{\frac{1}{6}}\right|^{2}\right)^{\frac{1}{2}} \leq\left(1+\left(\frac{3}{4}\right)^{\frac{1}{12}}\right)^{-1}\left(1-\left(\frac{3}{4}\right)^{\frac{1}{6}}\right):=a_{1}, \\
& \left|\sqrt{N^{-}}-\left(\sum_{j=1}^{N^{-}}\left(c_{j}^{-}\right)^{\frac{1}{6}}\right)^{\frac{1}{2}}\right|=\left|\left(\sum_{j=1}^{N^{-}} 1\right)^{\frac{1}{2}}-\left(\sum_{j=1}^{N^{-}}\left(c_{j}^{-}\right)^{\frac{1}{6}}\right)^{\frac{1}{2}}\right| \leq\left(\sum_{j=1}^{N^{-}}\left|1-\left(c_{j}^{-}\right)^{\frac{1}{12}}\right|^{2}\right)^{\frac{1}{2}} \\
& \leq\left(1+\left(\frac{4}{5}\right)^{\frac{1}{6}}\right)^{-1}\left(\sum_{j=1}^{N^{-}}\left|1-\left(c_{j}^{-}\right)^{\frac{1}{6}}\right|^{2}\right)^{\frac{1}{2}} \leq\left(1+\left(\frac{4}{5}\right)^{\frac{1}{6}}\right)^{-1} 5^{\frac{1}{2}}\left(\frac{3}{35}\right)^{\frac{3}{16}}\left(1-\left(\frac{3}{4}\right)^{\frac{1}{6}}\right):=a_{2} .
\end{aligned}
$$

Thus, by (2.2),

and so by explicit computations,

$$
\left|\sqrt{N}-\sqrt{N^{-}}\right| \leq a_{1}+a_{2}:=a
$$

$$
\left|N^{-}-N\right| \leq 2 a \sqrt{N}+a^{2} \leq\left(2 a+\frac{a^{2}}{\sqrt{2}}\right) \sqrt{N}<\frac{\sqrt{N}}{8} .
$$

In particular, if $N \leq 64$, then $N^{-}=N$.

2.3. Rigidity result for two solitons. In the case of an ingoing 2-soliton, we prove an unconditional result. For any $0<c<1$, we claim that if the ingoing 2 -soliton is also an outgoing $N$-soliton, then $N=2$ and the speeds at $+\infty$ and $-\infty$ are the same. In particular, it is a symmetric 2 -soliton. This result is not needed for the proof of Theorem 1.1 but it is proved for its own interest. Such question remains open for $N \geq 3$.

Proposition 2.2 (Rigidity of 2 -solitons). Let $0<c<1$. Let $u(t)$ be the outgoing 2-soliton of (1.1) satisfying

$$
\lim _{t \rightarrow+\infty}\left\|u(t)-Q(.-t)-Q_{c}(.-c t)\right\|_{H^{1}}=0 .
$$


Assume that $u(t)$ is an ingoing multi-soliton, i.e. there exist $0<c_{N}<\ldots<c_{1}$, and $\Delta_{1}, \ldots, \Delta_{N}$ such that

$$
\lim _{t \rightarrow-\infty}\left\|u(t)-\sum_{j=1}^{N} Q_{c_{j}}\left(.-c_{j} t-\Delta_{j}\right)\right\|_{H^{1}}=0 .
$$

Then,

(i) $u(t)$ is a pure 2-soliton,

$$
N=2 \quad \text { and } \quad c_{1}=1, \quad c_{2}=c .
$$

(ii) There exist $T_{0}, Y_{0} \in \mathbb{R}$ such that

$$
u(t, x)=u\left(-t+T_{0},-x+Y_{0}\right) .
$$

Let $u(t)$ be a solution of (1.1) as in the statement of Proposition 2.2. Property (ii) is a direct consequence of (i) and the uniqueness result in [11].

We now prove (i). By Lemma 2.1, we have

$$
1+c^{\frac{1}{6}}=\sum_{j=1}^{N} c_{j}^{\frac{1}{6}}, \quad 1+c^{\frac{7}{6}}=\sum_{j=1}^{N} c_{j}^{\frac{1}{6}}, \quad 1+c^{-\frac{1}{6}}=\sum_{j=1}^{N} c_{j}^{-\frac{1}{6}} .
$$

Setting $a_{j}=c_{j}^{\frac{1}{6}}$ and $x=c^{\frac{1}{6}}$, Proposition 2.2 (i) follows from the following elementary result.

Lemma 2.3. Let $0<x<1, N \geq 2$ and $0<a_{N}<\ldots<a_{1}$ be such that

$$
\sum_{j=1}^{N} a_{j}=1+x, \quad \sum_{j=1}^{N} a_{j}^{7}=1+x^{7}, \quad \sum_{j=1}^{N} \frac{1}{a_{j}}=1+\frac{1}{x} .
$$

Then, $N=2, a_{1}=1$ and $a_{2}=x$.

Proof. The case $N=2$ is easily treated. Let $a_{1}=a$ and $a_{2}=b, 0<b<a$ be such that

$$
\begin{aligned}
& a+b=1+x, \\
& a^{7}+b^{7}=1+x^{7}=1+(a+b-1)^{7} .
\end{aligned}
$$

Of course, $a=1, b=x$ is a solution. For $0<a<1+x, a \neq 1$, set $f(b)=$ $a^{7}+b^{7}-1-(a+b-1)^{7}$. We see that $f(1)=0$. Moreover, $f^{\prime}(b)=7\left(b^{6}-(a+b-1)^{6}\right)$, and thus $f^{\prime}(b)$ has no zero on $(0,1)$. It follows that $f$ has no zero on $[0,1)$ and so there are no other solution than $a=1, b=x$ for $N=2$.

We now consider the case $N \geq 3$. We define the bounded set

$$
\Omega=\left\{\left(a_{1}, \ldots, a_{N}\right) \in\left(\mathbb{R}_{+}^{*}\right)^{N} \mid \sum_{j=1}^{N} a_{j}=1+x, \sum_{j=1}^{N} a_{j}^{7}=1+x^{7}\right\} .
$$

and we look for the minimum on $\Omega$ of the following positive function $F$ :

$$
F\left(a_{1}, \ldots, a_{N}\right)=\sum_{j=1}^{N} \frac{1}{a_{j}} .
$$

Since $\lim _{a_{j} \rightarrow 0^{+}} F\left(a_{1}, \ldots, a_{N}\right)=+\infty, F$ reaches it minimum on $\Omega$. 
Note that if $\left(a_{1}, \ldots, a_{N}\right)$ is a point of $\Omega$ where the gradients of the functions $\sum_{j=1}^{N} a_{j}$ and $\sum_{j=1}^{N} a_{j}^{7}$ are colinear,then $a_{j}=a$ for all $j \in\{1, \ldots, N\}$. Thus,

$$
N a=1+x, \quad N a^{7}=1+x^{7} .
$$

It follows that $\frac{1}{N}<a<\frac{2}{N}$ and $a^{6}=\frac{1+x^{7}}{1+x}>\frac{1}{2}$ which imply $\left(\frac{2}{N}\right)^{6} \geq \frac{1}{2}$ and so $N \leq 2^{1+\frac{1}{6}}$. This is a contradiction and so no such point exists on $\Omega$.

Therefore, we can apply the method of Lagrange multipliers to characterize extrema of $F$ on $\Omega$. For a critical point $\left(a_{1}, \ldots, a_{N}\right) \in \Omega$ of $F$, there exist $\lambda, \mu \in \mathbb{R}$ such that

$$
\forall j=1, \ldots, N, \quad \frac{1}{a_{j}^{2}}=\lambda+\mu a_{j}^{6} .
$$

Let $g(\alpha)=\mu \alpha^{4}+\lambda \alpha-1$. We see that $g^{\prime}$ has at most one root on $[0,+\infty)$ and $g$ has at most two roots on $[0,+\infty)$. We have already observe that $\Omega$ contains no point of the form $(a, \ldots, a)$.

Therefore, the $\left(a_{j}\right)$ take exactly two different values: there exist $0<b<a$ and $1 \leq k<N$ such that

$$
\begin{aligned}
& k a+(N-k) b=1+x, \\
& k a^{7}+(N-k) b^{7}=1+x^{7} .
\end{aligned}
$$

- For $1 / 3 \leq x<1$ : Note that for all $y>0, \frac{1}{y} \geq 2-y$. Thus,

$$
\frac{k}{a}+\frac{N-k}{b} \geq 2 N-k a-(N-k) b .
$$

Since

we find

$$
k a+(N-k) b=1+x,
$$

$$
\frac{k}{a}+\frac{N-k}{b} \geq 2 N-1-x>4 \geq 1+\frac{1}{x} .
$$

It follows that at such a critical point, $F$ is strictly greater that $1+\frac{1}{x}$.

- For $k=1,0<x<1 / 3$. Then,

$$
\begin{aligned}
& a-1+(N-1) b=x, \\
& a^{7}-1+(N-1) b^{7}=x^{7} .
\end{aligned}
$$

Since

$$
\left|a^{7}-1\right|=|a-1|\left(a^{6}+a^{5}+a^{4}+a^{3}+a^{2}+a+1\right) \geq|a-1|,
$$

we obtain from the second identity

$$
|a-1| \leq x^{7}+(N-1) b^{7} .
$$

Combining this with the first identity and then using $b<\frac{2}{3}$ (since $3 b<a+2 b \leq$ $1+x<2$ ), we get

$$
(N-1) b<x+x^{7}+(N-1) b^{7}<\frac{11}{10} x+\frac{N-1}{10} b,
$$

and so $\frac{9}{11}(N-1) b<x$. In particular

$$
\frac{N-1}{b} \geq \frac{9}{11} \frac{(N-1)^{2}}{x} \geq \frac{9}{11} \frac{4}{x}>1+\frac{1}{x}
$$


Again, at such a critical point, $F$ is strictly greater that $1+\frac{1}{x}$.

- For $2 \leq k \leq N-1,0<x<1 / 3$. Since $0<b<a$ and

$$
N b<k a+(N-k) b=1+x,
$$

we have

$$
a \leq \frac{1+x}{k}, \quad b \leq \frac{1+x}{N} .
$$

Thus,

$$
1+x^{7}=k a^{7}+(N-k) b^{7} \leq\left(k^{-6}+N^{-6}\right)(1+x)^{7} \leq\left(2^{-6}+3^{-6}\right)\left(\frac{4}{3}\right)^{7}<1,
$$

a contradiction. This means that no such critical point exist in this case.

\section{Pointwise decay estimates for ingoing multisoliton}

This section is devoted to the proof of Lemma 1.4 by standard monotonicity arguments (see e.g. [13], [14] and [5]).

\subsection{Monotonicity result. Set}

$$
\phi(x)=\frac{2}{\pi} \arctan (\exp x), \quad \phi^{\prime}(x)=\frac{1}{\pi \cosh x},
$$

so that

$$
\phi^{\prime \prime \prime} \leq \phi^{\prime} \text { on } \mathbb{R} .
$$

Let us recall the following result (see [14]) whose proof is given in Appendix A for the sake of completeness.

Lemma 3.1 (Mass-energy monotonicity). Let $0<\sigma<\sigma^{\prime}<c_{0}$ and $C_{0}>0$. There exists $\alpha_{0}>0$ such that the following holds. Let $u(t)$ be a solution of (1.1) such that there exists $R>1$ with

$$
\forall t \in\left[t_{1}, t_{2}\right], \quad\|u(t)\|_{H^{1}} \leq C_{0}, \quad\|u(t)\|_{L^{2}\left(x>c_{0} t+R\right)} \leq \alpha_{0} .
$$

Then, there exists $C=C\left(\sigma, \sigma^{\prime}, c_{0}, C_{0}, R\right)>0$ such that, for all $x_{0}>0$,

$$
\begin{aligned}
& \int\left(u_{x}^{2}+u^{2}\right)\left(t_{2}, x\right) \phi\left(\sqrt{\sigma}\left(x-c_{0} t_{2}-x_{0}\right)\right) d x \\
& \leq 2 \int\left(u_{x}^{2}+u^{2}\right)\left(t_{1}, x\right) \phi\left(\sqrt{\sigma}\left(x-c_{0} t_{1}-\left(c_{0}-\sigma^{\prime}\right)\left(t_{2}-t_{1}\right)-x_{0}\right)\right) d x+C e^{-\sqrt{\sigma} x_{0}} .
\end{aligned}
$$

3.2. Decay on the right. Proof of Lemma 1.4. Step 1. Decay at $t=0$. Let

$0<\sigma_{1}<c_{N}^{-}$. We claim that there exists $C>0$ such that

$$
\forall x_{0}>0, \quad \int\left(u_{x}^{2}+u^{2}\right)(0, x) \phi\left(\sqrt{\sigma_{1}}\left(x-x_{0}\right)\right) d x \leq C e^{-\sqrt{\sigma_{1}} x_{0}} .
$$


Let $\sigma=\sigma_{1}, \sigma^{\prime}=\frac{1}{2}\left(\sigma_{1}+c_{N}^{-}\right), c_{0}=c_{N}^{-}$and $C_{0}=\sup _{t}\|u(t)\|_{H^{1}}$. Let $\alpha_{0}$ be given by Lemma 3.1. From (1.12), for $t_{0}>0$ large enough, for all $t<-t_{0}$, for all $x$,

$$
\begin{aligned}
|u(t, x)| & \leq \sum_{j}\left|Q_{c_{j}}\left(x-c_{j} t-\Delta_{j}\right)\right|+\left\|u(t)-\sum_{j} Q_{c_{j}}\left(.-c_{j} t-\Delta_{j}\right)\right\|_{H^{1}} \\
& \leq C \sum_{j=1}^{N} e^{-\sqrt{c_{j}}\left(x-c_{j} t\right)}+\frac{1}{2} \alpha_{0} .
\end{aligned}
$$

Thus, there exists $R>0$ such that

$$
\sup _{t \leq-t_{0}}\|u(t)\|_{L^{2}\left(x>c_{N}^{-} t+R\right)}<\alpha_{0} .
$$

By possibly taking a larger $R$, we also have

$$
\sup _{t \leq 0}\|u(t)\|_{L^{2}\left(x>c_{N}^{-} t+R\right)}<\alpha_{0} .
$$

Applying Lemma 3.1 on $[t, 0]$, for any $t<0$, for all $x_{0}>0$,

$$
\begin{aligned}
& \int\left(u_{x}^{2}+u^{2}\right)(0, x) \phi\left(\sqrt{\sigma_{1}}\left(x-x_{0}\right)\right) d x \\
& \leq 2 \int\left(u_{x}^{2}+u^{2}\right)(t, x) \phi\left(\sqrt{\sigma_{1}}\left(x-x_{0}-\sigma^{\prime} t\right)\right) d x+C e^{-\sqrt{\sigma_{1}} x_{0}} .
\end{aligned}
$$

By (1.12) and the definition of $\phi$,

$$
\lim _{t \rightarrow-\infty} \int\left(u_{x}^{2}+u^{2}\right)(t, x) \phi\left(\sqrt{\sigma_{1}}\left(x-x_{0}-\sigma^{\prime} t\right)\right) d x=0,
$$

and (3.4) follows.

Step 2. Decay on the right for $t>0$. Let $\sigma_{1} \leq \sigma_{2}<\sigma_{3}<1$. We claim

$$
\forall x>0, \forall t>0, \quad|u(t, t+x)| \leq C\left(e^{-\frac{1}{2} \sqrt{\sigma_{1}}\left(x+\left(1-\sigma_{3}\right) t\right)}+e^{-\frac{1}{2} \sqrt{\sigma_{2}} x}\right) .
$$

Let $\sigma=\sigma_{2}, \sigma^{\prime}=\sigma_{3}, c_{0}=1$ and $C_{0}=\sup _{t}\|u(t)\|_{H^{1}}$. Let $\alpha_{0}$ be given by Lemma 3.1. As before, from (1.12), there exists $R>0$ such that

$$
\sup _{t \geq 0}\|u(t)\|_{L^{2}(x \geq t+R)}<\alpha_{0} .
$$

Applying Lemma 3.1, for $t>0, x_{0}>0$,

$$
\begin{aligned}
& \int\left(u_{x}^{2}+u^{2}\right)(t, x) \phi\left(\sqrt{\sigma_{2}}\left(x-t-x_{0}\right)\right) d x \\
& \leq 2 \int\left(u_{x}^{2}+u^{2}\right)(0, x) \phi\left(\sqrt{\sigma_{2}}\left(x-\left(1-\sigma_{3}\right) t-x_{0}\right)\right) d x+C e^{-\sqrt{\sigma_{2}} x_{0}} .
\end{aligned}
$$

Recall (3.4),

$$
\forall x_{0}>0, \quad \int\left(u_{x}^{2}+u^{2}\right)(0, x) \phi\left(\sqrt{\sigma_{1}}\left(x-x_{0}\right)\right) d x \leq C e^{-\sqrt{\sigma_{1}} x_{0}} .
$$


Since $\phi \geq \frac{1}{2}$ on $\mathbb{R}^{+}$and since for all $y \in \mathbb{R}, \phi\left(\sqrt{\sigma_{2}} y\right) \leq 2 \phi\left(\sqrt{\sigma_{1}} y\right)$ (by $\left.\sigma_{2} \geq \sigma_{1}\right)$, we obtain

$$
\begin{aligned}
& \int_{x>t+x_{0}}\left(u_{x}^{2}+u^{2}\right)(t, x) d x \leq 2 \int\left(u_{x}^{2}+u^{2}\right)(t, x) \phi\left(\sqrt{\sigma_{2}}\left(x-t-x_{0}\right)\right) d x \\
& \leq 4 \int\left(u_{x}^{2}+u^{2}\right)(0, x) \phi\left(\sqrt{\sigma_{2}}\left(x-\left(1-\sigma_{3}\right) t-x_{0}\right)\right) d x+C e^{-\sqrt{\sigma_{2}} x_{0}} \\
& \leq 8 \int\left(u_{x}^{2}+u^{2}\right)(0, x) \phi\left(\sqrt{\sigma_{1}}\left(x-\left(1-\sigma_{3}\right) t-x_{0}\right)\right) d x+C e^{-\sqrt{\sigma_{2}} x_{0}} \\
& \leq C e^{-\sqrt{\sigma_{1}}\left(x_{0}+\left(1-\sigma_{3}\right) t\right)}+C e^{-\sqrt{\sigma_{2}} x_{0}} .
\end{aligned}
$$

Estimate (3.5) then follows from

$$
\|u\|_{L^{\infty}\left(x>t+x_{0}\right)}^{2} \leq 2\left\|u_{x}\right\|_{L^{2}\left(x>t+x_{0}\right)}\|u\|_{L^{2}\left(x>t+x_{0}\right)} \leq\left\|u_{x}\right\|_{L^{2}\left(x>t+x_{0}\right)}^{2}+\|u\|_{L^{2}\left(x>t+x_{0}\right)}^{2} .
$$

Step 3. End of the proof of Lemma 1.4. We first claim the following technical facts.

\section{Claim 1.}

$$
\begin{gathered}
\frac{\sigma_{0}}{\gamma_{0}} \geq \sqrt{c_{j_{0}+1} c_{j_{0}}} . \\
\frac{4 \sigma_{0}}{\frac{\sigma_{0}}{\gamma_{0}}-\left(1-c_{j_{0}}\right)}<1, \\
\frac{\sigma_{0}}{\gamma_{0}}+c_{j_{0}}-\left(\max \left(\frac{4}{5}, \frac{4 \sigma_{0}}{\frac{\sigma_{0}}{\gamma_{0}}-\left(1-c_{j_{0}}\right)}\right)\right)^{2}>5 \sigma_{0} .
\end{gathered}
$$

Assume Claim 1, Let

$$
\begin{gathered}
\sigma_{1}=\frac{16}{25}<c_{N}^{-} \quad \text { by Lemma 1.3. } \\
\sigma_{2}=\left(\max \left(\frac{4}{5}, \frac{4 \bar{\sigma}_{0}}{\frac{\sigma_{0}}{\gamma_{0}}-\left(1-c_{j_{0}}\right)}\right)\right)^{2}<1, \quad \sigma_{1} \leq \sigma_{2}<\sigma_{3}<1, \quad \bar{\sigma}_{0}>\sigma_{0},
\end{gathered}
$$

where by (3.8) and by continuity, we fix $\bar{\sigma}_{0}>\sigma_{0}$ close enough to $\sigma_{0}$ and $\sigma_{3}>\sigma_{2}$ close enough to $\sigma_{2}$ so that

$$
\frac{\sigma_{0}}{\gamma_{0}}+c_{j_{0}}-\sigma_{3}>5 \bar{\sigma}_{0}
$$

Applying (3.5) with $x=x_{0}(t)-t$ where $x_{0}(t)=\left(\frac{\sigma_{0}}{\gamma_{0}}+c_{j_{0}}\right) t-K_{0}$, we obtain using (3.9),

$$
\begin{aligned}
\left|u\left(t, x_{0}(t)\right)\right| & \leq C e^{-\frac{2}{5}\left(x_{0}(t)-\sigma_{3} t\right)}+C e^{-\frac{1}{2} \sqrt{\sigma_{2}}\left(x_{0}(t)-t\right)} \\
& \leq C e^{-\frac{2}{5}\left[\left(\frac{\sigma_{0}}{\gamma_{0}}+c_{j_{0}}-\sigma_{3}\right) t-K_{0}\right]}+C e^{-\frac{1}{2} \sqrt{\sigma_{2}}\left[\left(\frac{\sigma_{0}}{\gamma_{0}}+c_{j_{0}}-1\right) t-K_{0}\right]} \\
& \leq C\left(K_{0}\right) e^{-2 \bar{\sigma}_{0} t} \leq e^{-2 \sigma_{0} t},
\end{aligned}
$$

for all $t>t_{0}\left(K_{0}\right)$, provided $t_{0}\left(K_{0}\right)$ is large enough.

Proof of Claim 1, First, by explicit computations, we see that

$$
0 \leq c \leq 1 \Rightarrow \sqrt{1-\frac{3}{4}} c \geq 1-\frac{\sqrt{c}}{2},
$$


so that $\gamma(c):=\sqrt{1-\frac{3}{4} c}-\frac{\sqrt{c}}{2} \geq 1-\sqrt{c}$. Moreover,

$$
\gamma(c)=\frac{1-c}{\sqrt{1-\frac{3}{4} c}+\frac{\sqrt{c}}{2}} \leq 1-c,
$$

thus

$$
\forall c \in[0,1], \quad 1-\sqrt{c} \leq \sqrt{1-\frac{3}{4} c}-\frac{\sqrt{c}}{2} \leq 1-c .
$$

Since $\gamma_{0}=\sqrt{c_{j_{0}}} \gamma\left(c_{j_{0}+1} / c_{j_{0}}\right)$, we obtain

$$
\frac{\sigma_{0}}{\gamma_{0}} \geq \frac{\sqrt{c_{j_{0}+1}}\left(c_{j_{0}}-c_{j_{0}+1}\right)}{\sqrt{c_{j_{0}}}\left(1-c_{j_{0}+1} / c_{j_{0}}\right)}=\sqrt{c_{j_{0}+1} c_{j_{0}}} .
$$

Next, we prove (3.7). Observe that

$$
\sigma_{0}=c_{j_{0}} \gamma_{0}-\gamma_{0}^{3},
$$

and, since $\frac{3}{4}<c_{j} \leq 1$ for all $j$,

$$
0<\gamma_{0}<\sqrt{c_{j_{0}}}\left(\sqrt{1-\frac{9}{16}}-\frac{\sqrt{3}}{4}\right)=\frac{\sqrt{c_{j_{0}}}}{4}(\sqrt{7}-\sqrt{3}) .
$$

Since $1-c_{j_{0}+1}=1-c_{j_{0}}+c_{j_{0}}-c_{j_{0}+1} \leq \frac{1}{4}$, we obtain

$$
\begin{aligned}
4 \sigma_{0} & =4 \sqrt{c_{j_{0}+1}}\left(c_{j_{0}}-c_{j_{0}+1}\right) \leq 4 \sqrt{c_{j_{0}+1}}\left(\frac{1}{4}-\left(1-c_{j_{0}}\right)\right) \\
& \leq \sqrt{c_{j_{0}+1}}-4 \sqrt{c_{j_{0}+1}}\left(1-c_{j_{0}}\right) \leq c_{j_{0}} \sqrt{c_{j_{0}+1}}-3 \sqrt{c_{j_{0}+1}}\left(1-c_{j_{0}}\right) \\
& \leq c_{j_{0}} \sqrt{c_{j_{0}+1}}-3 \frac{\sqrt{3}}{2}\left(1-c_{j_{0}}\right) \leq \sqrt{c_{j_{0}+1}} \sqrt{c_{j_{0}}}-\left(1-c_{j_{0}}\right) \leq \frac{\sigma_{0}}{\gamma_{0}}-\left(1-c_{j_{0}}\right) .
\end{aligned}
$$

Finally, we prove (3.8). We begin with the case where $\frac{\sigma_{0}}{\frac{\sigma_{0}}{\gamma_{0}}-\left(1-c_{j_{0}}\right)}<\frac{1}{5}$. Then, necessarily $\gamma_{0}<\frac{1}{5}$. It is then clear that, using (3.12),

$$
\frac{\sigma_{0}}{\gamma_{0}}+c_{j_{0}}-\frac{16}{25}-5 \sigma_{0}=c_{j_{0}}-\frac{16}{25}+\left(c_{j_{0}}-\gamma_{0}^{2}\right)\left(1-5 \gamma_{0}\right) \geq 0 .
$$

Second, we assume $\frac{\sigma_{0}}{\frac{\sigma_{0}}{\gamma_{0}}-\left(1-c_{j_{0}}\right)} \geq \frac{1}{5}$. We distinguish two cases depending on the value of $j_{0}$.

If $j_{0}=1$ then $c_{j_{0}}=1$ and we are reduced to prove

$$
\frac{\sigma_{0}}{\gamma_{0}}+1-16 \gamma_{0}^{2}>5 \sigma_{0}
$$

where $\frac{\sigma_{0}}{\gamma_{0}}=1-\gamma_{0}^{2}$, so that it is sufficient to have

$$
0<\gamma_{0} \leq \frac{\sqrt{7}-\sqrt{3}}{4} \Rightarrow 2-5 \gamma_{0}-17 \gamma_{0}^{2}+5 \gamma_{0}^{3}>0
$$

which is easily checked by explicit computations.

We now consider the case where $j_{0} \geq 2$ and thus $N \geq 3$. Let

$$
\tilde{\sigma}_{0}=\frac{\sigma_{0}}{c_{j_{0}}^{\frac{3}{2}}}, \quad \tilde{\gamma}_{0}=\frac{\gamma_{0}}{c_{j_{0}}^{\frac{1}{2}}}, \quad \frac{\tilde{\sigma}_{0}}{\tilde{\gamma}_{0}}=1-\tilde{\gamma}_{0}^{2}
$$


Then, (3.8) is equivalent to

$$
\frac{\tilde{\sigma}_{0}}{\tilde{\gamma}_{0}}+1-\left(\frac{4 \tilde{\sigma}_{0}}{\frac{\tilde{\sigma}_{0}}{\tilde{\gamma}_{0}}-\left(\frac{1}{c_{j_{0}}}-1\right)}\right)^{2}>5 \sqrt{c_{j_{0}}} \tilde{\sigma}_{0} .
$$

It is clear that the following inequality

$$
\frac{\tilde{\sigma}_{0}}{\tilde{\gamma}_{0}}+1-\left(\frac{4 \tilde{\sigma}_{0}}{\frac{\tilde{\sigma}_{0}}{\tilde{\gamma}_{0}}-\frac{1}{3}}\right)^{2}>5 \tilde{\sigma}_{0} .
$$

would imply (3.15). By explicit computations, one can see that (3.16) is not satisfied for all $\tilde{\gamma}_{0} \in\left(0, \frac{\sqrt{7}-\sqrt{3}}{4}\right]$. At this point, we need to use the definition of $j_{0}$ and the fact that $j_{0} \geq 2$ to lower the value of $\tilde{\gamma}_{0}$ for which we have to check (3.16).

Indeed, by the definition of $j_{0}$, we have

$$
\frac{\sqrt{3}}{2}\left(c_{j_{0}}-c_{j_{0}+1}\right) \leq \sqrt{c_{j_{0}+1}}\left(c_{j_{0}}-c_{j_{0}+1}\right) \leq \sqrt{c_{2}}\left(1-c_{2}\right) \leq\left(1-c_{2}\right) .
$$

Since $1-c_{2}+c_{j_{0}}-c_{j_{0}+1} \leq \frac{1}{4}$, we obtain $c_{j_{0}}-c_{j_{0}+1} \leq \frac{1}{2(2+\sqrt{3})}$ and so by simple computations,

$$
\frac{c_{j_{0}+1}}{c_{j_{0}}} \geq \frac{1}{1+\frac{2}{3(2+\sqrt{3})}} \quad \text { which implies } \quad \tilde{\gamma}_{0} \leq \frac{3}{20} .
$$

Moreover, we easily check that (3.16) is indeed satisfied for all $\tilde{\gamma}_{0} \in\left[0, \frac{3}{20}\right]$.

\section{Construction and lower bound of the approximate solution}

This section contains the main ingredient of the proof, i.e. the construction of an approximate solution for $t \gg 1$, and the description of its asymptotics for $x \gg 1$. The approximate solution is built by explicit resolution of the main contribution of the interactions of the solitons for $t \rightarrow+\infty$, i.e. where solitons are decoupled. Note that such refined computations were not needed for existence result.

Recall the following notation. Let $j_{0} \in\{1, \ldots, N-1\}$ be such that

$$
\sqrt{c_{j_{0}+1}}\left(c_{j_{0}}-c_{j_{0}+1}\right)=\min _{j} \sqrt{c_{j+1}}\left(c_{j}-c_{j+1}\right):=\sigma_{0}
$$

and

$$
\gamma_{0}=\sqrt{c_{j_{0}}-\frac{3}{4} c_{j_{0}+1}}-\frac{1}{2} \sqrt{c_{j_{0}+1}}, \quad x_{0}(t)=\left(\frac{\sigma_{0}}{\gamma_{0}}+c_{j_{0}}\right) t-K_{0}
$$

where $K_{0}>0$.

Proposition 4.1 (Approximate solution). Assume (1.10). There exists a function $V(t, x)$ such that

1. There exists $C>0$ such that, for all $t \geq 0$,

$$
\left\|V(t)-\sum_{j=1}^{N} Q_{c_{j}}\left(x-c_{j} t-\Delta_{j}\right)\right\|_{H^{3}} \leq C e^{-\sigma_{0} t} .
$$

2. For all $t \geq 0$,

$$
\left\|\partial_{t} V+\partial_{x}\left(\partial_{x}^{2} V+V^{4}\right)\right\|_{H^{3}} \leq C e^{-2 \sigma_{0} t}
$$


3. There exist $\kappa>0$ and $t_{1}=t_{1}\left(K_{0}\right)>0$, such that, for all $t \geq t_{1}$,

$$
\left|V\left(t, x_{0}(t)\right)\right| \geq \kappa e^{\gamma_{0} K_{0}} e^{-2 \sigma_{0} t} .
$$

This section is devoted to the proof of Proposition 4.1,

4.1. ODE analysis. For $c>0$, let

$$
\begin{gathered}
L_{c} f=-f^{\prime \prime}+c f-4 Q_{c}^{3} f, \quad L=L_{1}, \\
\Lambda Q_{c}=\frac{1}{c}\left(\frac{1}{3} Q_{c}+\frac{1}{2} x Q_{c}^{\prime}\right)=\frac{d}{d c^{\prime}} Q_{c^{\prime} \mid c^{\prime}=c}, \quad \Lambda Q=\Lambda Q_{1}, \quad L \Lambda Q=-Q .
\end{gathered}
$$

Denote the $L^{2}$ scalar product by $(f, g)=\int f(x) g(x) d x$.

Following the outline of the proof in the Introduction, we define the solution of equation (1.21) related to the main perturbative terms in the construction of the approximate solution.

Lemma 4.2. Let $\frac{3}{4} \leq c<1$. There exists a unique solution $A_{c} \in H^{10}$ of

$$
\left(L A_{c}\right)^{\prime}+\sqrt{c}(1-c) A_{c}=G_{c}^{\prime} \text { where } G_{c}(x)=e^{-\sqrt{c} x} Q^{3}(x) .
$$

Moreover, $A_{c}$ satisfies the following, for all $k \geq 0$,

(i) Decay estimates.

$$
\left|A_{c}^{(k)}(x)\right| \lesssim e^{\gamma_{c}^{0} x} \quad \text { for } x<0, \quad\left|A_{c}^{(k)}(x)\right| \lesssim e^{-\gamma_{c}^{\mathrm{I}} x} \quad \text { for } x>0,
$$

where

$$
\begin{gathered}
\gamma_{c}^{0}=\sqrt{1-\frac{3}{4}} c+\frac{1}{2} \sqrt{c}, \quad \gamma_{c}^{\mathrm{I}}=\sqrt{1-\frac{3}{4}} c-\frac{1}{2} \sqrt{c}, \quad \gamma_{c}^{\mathrm{II}}=\sqrt{c}, \\
0<\gamma_{c}^{\mathrm{I}}<\gamma_{c}^{\mathrm{II}}=\sqrt{c}<\gamma_{c}^{0}=\gamma_{c}^{\mathrm{I}}+\gamma_{c}^{\mathrm{II}} .
\end{gathered}
$$

(ii) Asymptotics at $+\infty$. There exist $a_{c}^{\mathrm{I}, \mathrm{II}} \in \mathbb{R}$ such that, for all $k \geq 0$,

$$
\left|(d / d x)^{k}\left(A_{c}(x)-a_{c}^{\mathrm{I}} e^{-\gamma_{c}^{\mathrm{I}} x}-a_{c}^{\mathrm{II}} e^{-\gamma_{c}^{\mathrm{II}} x}\right)\right| \lesssim e^{-\frac{3}{2}|x|} \quad \text { for } x>0 .
$$

(iii) Generic exponential decay on the right-hand side.

$$
a_{c}^{\mathrm{I}}=\lim _{x \rightarrow+\infty} A_{c}(x) e^{\gamma_{c}^{\mathrm{I}} x} \neq 0 .
$$

(iv) Continuity of $a_{c}^{\mathrm{I}}$. The function $c \in\left[\frac{3}{4}, 1\right) \mapsto a_{c}^{\mathrm{I}}$ is continuous. In particular, $a_{c}^{\mathrm{I}}$ has a constant sign on $\left[\frac{3}{4}, 1\right)$.

Remark 4.3. Note that $\gamma_{c}^{0},-\gamma_{c}^{\mathrm{I}}$ and $-\gamma_{c}^{\mathrm{II}}$ are the three roots of $\gamma^{3}-\gamma-\sqrt{c}(1-c)=$ $(\gamma+\sqrt{c})\left(\gamma^{2}-\sqrt{c} \gamma-(1-c)\right)=0$. Since $\frac{3}{4} \leq c<1$, we have

$$
\sqrt{c}<\gamma_{c}^{0}<2 \sqrt{c}, \quad 0<\gamma_{c}^{\mathrm{I}}<\sqrt{c} .
$$

It is a key point in our proof to be able to prove that $A_{c}$ has generic decay, i.e. the exponential decay $e^{-\gamma_{c}^{\mathrm{I}} x}$ for $x>0$.

For $0<c \leq \frac{1}{3}$, the situation is different since $\gamma_{c}^{\mathrm{I}} \geq \sqrt{c}$. In particular, $e^{-\sqrt{c} x}$ is the generic decay of $A_{c}$ in this case. The strategy of this paper cannot be applied directly.

Remark 4.4. By Claim 1

$$
1-\sqrt{c} \leq \gamma_{c}^{\mathrm{I}} \leq 1-c .
$$


Proof. Proof of (i)-(ii). First, note that for $0<\theta<\frac{2}{3 \sqrt{3}}$, equation $\gamma^{3}-\gamma-\theta=0$ has three distinct real roots: $\gamma_{\theta}^{0},-\gamma_{\theta}^{\mathrm{I}}$ and $-\gamma_{\theta}^{\mathrm{II}}$, where

$$
0 \leq \gamma_{\theta}^{\mathrm{I}}<\gamma_{\theta}^{\mathrm{II}}<1<\gamma_{\theta}^{0}=\gamma_{\theta}^{\mathrm{I}}+\gamma_{\theta}^{\mathrm{II}} \leq \frac{2}{\sqrt{3}}
$$

From the spectral analysis of Pego and Weinstein [27] and standard ODE arguments, one has the following general result.

Claim 2. (a) Existence. For all $\theta>0$, for all $F \in L^{2}$, there exists a unique solution $A \in H^{3}$ of

$$
(L A)^{\prime}+\theta A=F .
$$

(b) Decay. Let $F$ be a $C^{\infty}$ function such that

$$
\forall k \geq 0, \forall x \in \mathbb{R}, \quad\left|F^{(k)}(x)\right| \leq C_{k} e^{-\frac{3}{2}|x|} .
$$

Assume $0<\theta<\frac{2}{3 \sqrt{3}}$ and let $A$ be the solution of (4.12). Then, $A$ is $C^{\infty}$ and satisfies, for all $k \geq 0$,

- Decay estimates.

$$
\left|A^{(k)}(x)\right| \lesssim e^{\gamma_{\theta}^{0} x} \quad \text { for } x<0, \quad\left|A^{(k)}(x)\right| \lesssim e^{-\gamma_{\theta}^{\mathrm{I}} x} \quad \text { for } x>0 .
$$

- Asymptotics at $+\infty$. There exists $a_{\theta}^{\mathrm{I}, \mathrm{II}} \in \mathbb{R}$ such that

$$
\left|(d / d x)^{k}\left(A(x)-a_{\theta}^{\mathrm{I}} e^{-\gamma_{\theta}^{\mathrm{I}} x}-a_{\theta}^{\mathrm{II}} e^{-\gamma_{\theta}^{\mathrm{II}} x}\right)\right| \lesssim e^{-\frac{3}{2}|x|} \quad \text { for } x>0 .
$$

Proof of Claim 2. Proof of (a). In [27], it is proved that no real nonzero eigenvalue of the operator $(L A)^{\prime}$ exists. We reproduce the proof here for the sake of completeness. Recall the following basic facts on $L$ (see [34])

- $\operatorname{Ker} L=\left\{\lambda Q^{\prime}, \lambda \in \mathbb{R}\right\}$;

- There exists $\mu>0$ such that for any $f \in H^{1}$,

$$
(f, Q)=\left(f, Q^{\prime}\right)=0 \quad \Rightarrow \quad(L f, f)=\int\left(f^{\prime}\right)^{2}+f^{2}-5 Q^{4} f^{2} \geq \mu\|f\|_{H^{1}}^{2} .
$$

Let $A$ be a solution of $(L A)^{\prime}+\theta A=0$. Let $\bar{A}=A+a Q^{\prime}$, where $a$ is such that $\int \bar{A} Q^{\prime}=0$. Then, since $L Q^{\prime}=0$,

$$
(L \bar{A})^{\prime}+\theta \bar{A}=a \theta Q^{\prime}
$$

Taking the scalar product of (4.16) by $Q$, we obtain $(\bar{A}, Q)=0$. Moreover, taking the scalar product of (4.16) by $L \bar{A}$, we get $(L \bar{A}, \bar{A})=0$. By (4.15), it follows that $\bar{A}=0$, and so $A=-a Q^{\prime}$. But then $\theta A=0$, so that $\theta=0$ or $A \equiv 0$.

Once we know that no real nonzero eigenvalue exists, the invertibility of $(L A)^{\prime}+$ $\theta A$ follows from usual arguments (Fredholm alternative).

Proof of (b). These properties follow from standard ODE arguments.

Apply Claim 2 to $F=G_{c}^{\prime}$ and $\theta=\sqrt{c}(1-c)$. There exists a unique $A_{c} \in H^{3}$ such that $\left(L A_{c}\right)^{\prime}+\sqrt{c}(1-c) A_{c}=G_{c}^{\prime}$. Note that $\sqrt{c}(1-c)<\frac{2}{3 \sqrt{3}}$, and thus (i) and (ii) are direct consequences of Claim 2 and standard regularity arguments. 
Proof of (iii). This point is more delicate. Let $\frac{3}{4}<c<1$. Assume for the sake of the contradiction that $a_{c}^{\mathrm{I}}=0$, so that by (ii)

$$
\forall x>0, \quad\left|A_{c}^{(k)}(x)\right| \lesssim e^{-\sqrt{c}|x|} \lesssim e^{-\sqrt{\frac{3}{4}}|x|} .
$$

Step 1. Reduction to a dual equation. Let $a, b$ be such that $A=A_{c}-a \Lambda Q-b Q^{\prime}$ satisfies

$$
\int A Q^{\frac{5}{2}}=0, \quad \int A Q^{\prime}=0
$$

Note that $L Q^{\frac{5}{2}}=-\frac{21}{4} Q^{\frac{5}{2}}$ so that $a$ exists since $\left(Q^{\frac{5}{2}}, \Lambda Q\right)=-\frac{4}{21}\left(L Q^{\frac{5}{2}}, \Lambda Q\right)=$ $\frac{4}{21}\left(Q^{\frac{5}{2}}, Q\right) \neq 0$.

Then by $L \Lambda Q=-Q, A$ satisfies

$$
(L A)^{\prime}+\sqrt{c}(1-c) A+\sqrt{c}(1-c) a \Lambda Q-(a-\sqrt{c}(1-c) b) Q^{\prime}=G_{c}^{\prime} .
$$

Set $a_{0}=\sqrt{c}(1-c) a, B=-L A$. Then,

$$
L\left(B^{\prime}\right)+\sqrt{c}(1-c) B+a_{0} Q=-L\left(G_{c}^{\prime}\right), \quad \int B Q^{\frac{5}{2}}=\int B Q^{\prime}=0 .
$$

We decompose

$$
G_{c}=e^{-\sqrt{c} x} Q^{3}=G_{0}+\alpha Q^{\prime}+\beta Q,
$$

where

$$
\alpha=\frac{\int G_{c} Q^{\prime}}{\int\left(Q^{\prime}\right)^{2}} \neq 0, \quad \beta=\frac{\int G_{c} Q^{\frac{5}{2}}}{\int Q^{\frac{7}{2}}}, \quad \int G_{0} Q^{\frac{5}{2}}=\int G_{0} Q^{\prime}=0 .
$$

Now, we set $B_{0}=B+G_{0}$. We have thus proved

Claim 3. Assuming (4.17), there exists a smooth function $B_{0} \in H^{3}$ such that

$$
\begin{gathered}
L\left(B_{0}^{\prime}\right)+\sqrt{c}(1-c) B_{0}+a_{0} Q=-\alpha L\left(Q^{\prime \prime}\right)+\sqrt{c}(1-c) G_{0}, \\
\int B_{0} Q^{\frac{5}{2}}=\int B_{0} Q^{\prime}=0,
\end{gathered}
$$

$\forall k \geq 0, \forall x<0, \quad\left|B_{0}^{(k)}(x)\right| \lesssim e^{-\gamma_{c}^{0} x}, \quad \forall x>0,\left|B_{0}^{(k)}(x)\right| \lesssim e^{-\sqrt{\frac{3}{4}} x}$.

Step 2. We prove by Virial type arguments that such $B_{0}$ does not exist.

- Computation of $a_{0}$ and $\alpha$ from (4.20)-(4.21). On the one hand, we multiply (4.20) by $\Lambda Q$ and use $L \Lambda Q=-Q,\left(B_{0}, Q^{\prime}\right)=0,(Q, \Lambda Q)=\frac{1}{12} \int Q^{2}$, so that

$$
\sqrt{c}(1-c)\left(B_{0}, \Lambda Q\right)+\frac{a_{0}}{12} \int Q^{2}=-\alpha \int\left(Q^{\prime}\right)^{2}+\sqrt{c}(1-c) \int G_{0} \Lambda Q
$$

and thus (using $\left.\int\left(Q^{\prime}\right)^{2}=\frac{3}{7} \int Q^{2}\right)$

$$
a_{0}+\frac{36}{7} \alpha-\frac{12}{\int Q^{2}} \sqrt{c}(1-c)\left(G_{0}-B_{0}, \Lambda Q\right)=0 .
$$

On the other hand, consider $H_{0} \in L^{\infty}$ such that $H_{0}^{\prime} \in H^{2}, L H_{0}=1,\left(H_{0}, Q^{\prime}\right)=0$. An explicit expression of $H_{0}$ is available in [18], Claim 3.1:

$$
H_{0}=1+\frac{1}{3}\left(Q^{\prime} \int_{0}^{x} Q^{2}-2 Q^{3}\right) .
$$


Multiplying (4.20) by $H_{0}$ and using $\int B_{0}^{\prime}=\int Q^{\prime \prime}=0$, we find

$$
\sqrt{c}(1-c) \int B_{0} H_{0}+a_{0} \int Q H_{0}=\sqrt{c}(1-c) \int G_{0} H_{0},
$$

so that (note that $\int Q H_{0}=-\int(L \Lambda Q) H_{0}=-\int \Lambda Q=\frac{1}{6} \int Q$ ).

$$
a_{0}=\frac{6}{\int Q} \sqrt{c}(1-c)\left(G_{0}-B_{0}, H_{0}\right) .
$$

From (4.23) and (4.24), we deduce

$$
\alpha=-\frac{7}{3} \sqrt{c}(1-c)\left(G_{0}-B_{0}, J_{0}\right) \quad \text { where } \quad J_{0}=\frac{H_{0}}{2 \int Q}-\frac{\Lambda Q}{\int Q^{2}} .
$$

- Estimate on $B_{0}$ by Virial type identity. We adapt a strategy developed in [12], [16]. Multiply equation (4.20) by $B_{0} \frac{Q^{\prime}}{Q^{2}}$ and integrate. Note that all the integrals below are well-defined because of the decay properties (4.22). Then, using

$$
L\left(Q^{\prime \prime}\right)=\left(L Q^{\prime}\right)^{\prime}+4\left(Q^{3}\right)^{\prime} Q^{\prime}=12\left(Q^{\prime}\right)^{2} Q^{2},
$$

we have

$$
\begin{aligned}
& \int L\left(B_{0}^{\prime}\right) B_{0} \frac{Q^{\prime}}{Q^{2}}+\sqrt{c}(1-c) \int B_{0}^{2} \frac{Q^{\prime}}{Q^{2}}+a_{0} \int B_{0} \frac{Q^{\prime}}{Q} \\
& =-12 \alpha \int B_{0}\left(Q^{\prime}\right)^{3}+\sqrt{c}(1-c) \int B_{0} \frac{Q^{\prime}}{Q^{2}} G_{0} .
\end{aligned}
$$

The key argument to obtain a contradiction is a coercivity property of the quadratic form $\int L\left(B_{0}^{\prime}\right) B_{0} \frac{Q^{\prime}}{Q^{2}}$ under the orthogonality conditions (4.21).

\section{Claim 4.}

$$
\int L\left(B_{0}^{\prime}\right) B_{0} \frac{Q^{\prime}}{Q^{2}} \geq \frac{3}{8} \int \frac{B_{0}^{2}}{Q}+7 \int B_{0}^{2} Q^{2} .
$$

See proof of Claim 4 in Appendix B

Note that $\left(Q^{\prime}\right)^{2}=Q^{2}-\frac{2}{5} Q^{5} \leq Q^{2}$, so that $\frac{\left|Q^{\prime}\right|}{Q^{2}} \leq \frac{1}{Q}$, and thus for $\frac{3}{4} \leq c<1$, we have

where

$$
\int L\left(B_{0}^{\prime}\right) B_{0} \frac{Q^{\prime}}{Q^{2}}+\sqrt{c}(1-c) \int B_{0}^{2} \frac{Q^{\prime}}{Q^{2}} \geq \int B_{0}^{2} F_{0}
$$

$$
F_{0}=\frac{3-\sqrt{3}}{8} \frac{1}{Q}+7 Q^{2}
$$

Define

$$
N^{*}\left(B_{0}\right):=\left(\int B_{0}^{2} F_{0}\right)^{\frac{1}{2}}
$$

Define the operator $P$, the projection onto the orthogonal of $\operatorname{span}\left(Q^{\frac{5}{2}} ; Q^{\prime}\right)$ for the scalar product $\int\left(f g / F_{0}\right)$. In particular, for a given function $f$ such that $\int|f|^{2} Q<$ $+\infty$, we have

$$
\left|\int B_{0} f\right| \leq N^{*}\left(B_{0}\right) N(f) \text { where } \quad N(f)=\left(\int \frac{(P f)^{2}}{F_{0}}\right)^{\frac{1}{2}} .
$$

We claim 


\section{Claim 5.}

$$
\begin{aligned}
& N^{*}\left(B_{0}\right) \leq \sqrt{c}(1-c) \frac{k_{1}(c)}{k_{2}(c)}, \text { where } \\
& k_{1}(c)=N\left(-\frac{6}{\int Q}\left(G_{0}, H_{0}\right) \frac{Q^{\prime}}{Q}+28\left(G_{0}, J_{0}\right)\left(Q^{\prime}\right)^{3}+\frac{Q^{\prime}}{Q^{2}} G_{0}\right), \\
& k_{2}(c)=1-\sqrt{c}(1-c)\left(\frac{3}{\int Q} N\left(H_{0}\right) N\left(\frac{Q^{\prime}}{Q}\right)+14 N\left(J_{0}\right) N\left(\left(Q^{\prime}\right)^{3}\right)\right) .
\end{aligned}
$$

Proof of Claim 5. As a consequence of (4.26), (4.27), (4.24), (4.25), we get

$$
\begin{aligned}
N^{*}\left(B_{0}\right)^{2} & \leq-\frac{6}{\int Q} \sqrt{c}(1-c)\left(G_{0}-B_{0}, H_{0}\right)\left(B_{0}, \frac{Q^{\prime}}{Q}\right) \\
& +28 \sqrt{c}(1-c)\left(G_{0}-B_{0}, J_{0}\right)\left(B_{0},\left(Q^{\prime}\right)^{3}\right)+\sqrt{c}(1-c) \int B_{0} \frac{Q^{\prime}}{Q^{2}} G_{0} .
\end{aligned}
$$

Note that by parity properties

Thus,

$$
\begin{aligned}
& \left|\left(B_{0}, H_{0}\right)\left(B_{0}, \frac{Q^{\prime}}{Q}\right)\right| \leq \frac{1}{2} N^{*}\left(B_{0}\right)^{2} N\left(H_{0}\right) N\left(\frac{Q^{\prime}}{Q}\right), \\
& \left|\left(B_{0}, J_{0}\right)\left(B_{0},\left(Q^{\prime}\right)^{3}\right)\right| \leq \frac{1}{2} N^{*}\left(B_{0}\right)^{2} N\left(J_{0}\right) N\left(\left(Q^{\prime}\right)^{3}\right) .
\end{aligned}
$$

$$
\begin{aligned}
& N^{*}\left(B_{0}\right)\left[1-\sqrt{c}(1-c)\left(\frac{3}{\int Q} N\left(H_{0}\right) N\left(\frac{Q^{\prime}}{Q}\right)+14 N\left(J_{0}\right) N\left(\left(Q^{\prime}\right)^{3}\right)\right)\right] \\
& \leq \sqrt{c}(1-c) N\left(-\frac{6}{\int Q}\left(G_{0}, H_{0}\right) \frac{Q^{\prime}}{Q}+28\left(G_{0}, J_{0}\right)\left(Q^{\prime}\right)^{3}+\frac{Q^{\prime}}{Q^{2}} G_{0}\right),
\end{aligned}
$$

and (4.28) is proved.

- Conclusion. From (4.25) and Claim 5 ,

$$
\left|\frac{3}{7} \alpha+\sqrt{c}(1-c)\left(G_{0}, J_{0}\right)\right| \leq \sqrt{c}(1-c) N\left(J_{0}\right) N^{*}\left(B_{0}\right) \leq c(1-c)^{2} N\left(J_{0}\right) \frac{k_{1}(c)}{k_{2}(c)} .
$$

Thus,

$$
k(c):=\frac{c(1-c)^{2} N\left(J_{0}\right)}{\left|\frac{3}{7} \alpha+\sqrt{c}(1-c)\left(G_{0}, J_{0}\right)\right|} \frac{k_{1}(c)}{k_{2}(c)} \geq 1 .
$$

Observe that $k(c)$ is defined through explicit functions of $c$ (and does not depend on the function $B_{0}$ ). Therefore, one can compute $k(c)$ directly by various integrations of explicit functions. We check numerically that for all $c \in\left[\frac{3}{4}, 1\right], 0 \leq k(c) \leq 0.5<1$. In particular, a contradiction arises from (4.31) for all $c \in\left[\frac{3}{4}, 1\right]$ as desired.

Proof of (iv). Let $c, \tilde{c} \in\left(\frac{3}{4}, 1\right)$. Let $A_{c}, A_{\tilde{c}}$ be the corresponding solutions of (4.7).

First, we claim, for $C=C(c), \tilde{c}$ close to $c$,

$$
\left\|A_{c}-A_{\tilde{c}}\right\|_{H^{3}} \leq C|c-\tilde{c}| .
$$

Indeed, let $\epsilon=\sqrt{c}(1-c)-\sqrt{\tilde{c}}(1-\tilde{c}), G_{\epsilon}(x)=\left(e^{-\sqrt{c} x}-e^{-\sqrt{\tilde{c}} x}\right) Q^{3}(x)$. Then,

$$
\left(-L\left(A_{c}-A_{\tilde{c}}\right)\right)^{\prime}-\sqrt{\tilde{c}}(1-\tilde{c})\left(A_{c}-A_{\tilde{c}}\right)=\epsilon A_{c}+G_{\epsilon} .
$$


Let $A_{c}-A_{\tilde{c}}=D+a Q^{\prime}$ so that $\int D Q^{\prime}=0$ and

$$
(-L D)^{\prime}-\sqrt{\tilde{c}}(1-\tilde{c}) D=\epsilon A_{c}+G_{\epsilon}+a \sqrt{\tilde{c}}(1-\tilde{c}) Q^{\prime} .
$$

Multiplying the equation by $Q$ and integrating, we find $|(D, Q)| \leq C|c-\tilde{c}|$. Multiplying the equation by $L D$ and integrating, we find $|(L D, D)| \leq C|c-\tilde{c}|\|D\|_{L^{2}}$, so that by (4.15), $\|D\|_{H^{1}} \leq C|c-\tilde{c}|$, where $C$ depends on $c$. Multiplying the equation of $D$ by $Q^{\prime}$ and integrating, we find $|a| \leq C|c-\tilde{c}|$. Next, by the equation of $D$, $\|D\|_{H^{3}} \leq C|c-\tilde{c}|$, which implies $\left\|A_{c}-A_{\tilde{c}}\right\|_{H^{3}} \leq C|c-\tilde{c}|$.

Second, we set $a_{c}^{\mathrm{I}}=\lim _{x \rightarrow+\infty} A_{c}(x) e^{\gamma_{c}^{\mathrm{I}} x} \neq 0, a_{\tilde{c}}^{\mathrm{I}}=\lim _{x \rightarrow+\infty} A_{\tilde{c}}(x) e^{\gamma_{\tilde{c}}^{\mathrm{I}} x}$ and we prove that

$$
\lim _{\tilde{c} \rightarrow c} a_{\tilde{c}}^{\mathrm{I}}=a_{c}^{\mathrm{I}} .
$$

Let $0<\delta<\frac{1}{10}$ arbitrary. Fix $x_{0}>0$ large enough so that

$$
\begin{aligned}
& \sum_{j=0}^{2}\left|A_{c}^{(j)}\left(x_{0}\right)-\left(-\gamma_{c}^{\mathrm{I}}\right)^{j} a_{c}^{\mathrm{I}} e^{-\gamma_{c}^{\mathrm{I}} x_{0}}\right| \leq \delta e^{-\gamma_{c}^{\mathrm{I}} x_{0}}, \\
& \forall x>x_{0}, \quad Q^{2}(x) \leq \delta e^{-\frac{3}{2} x} .
\end{aligned}
$$

From (4.32) and continuity of $\gamma_{c}^{\mathrm{I}}$ in $c$, we take $|\tilde{c}-c|$ small enough so that

$$
\sum_{j=0}^{2}\left|A_{c}^{(j)}\left(x_{0}\right)-A_{\tilde{c}}^{(j)}\left(x_{0}\right)\right|+\left|e^{-\gamma_{c}^{\mathrm{I}} x_{0}}-e^{-\tilde{\gamma}_{c}^{\mathrm{I}} x_{0}}\right|+\left|\gamma_{c}^{\mathrm{I}}-\tilde{\gamma}_{c}^{\mathrm{I}}\right| \leq \delta e^{-\gamma_{c}^{\mathrm{I}} x_{0}} .
$$

Then by (4.35),

$$
\sum_{j=0}^{2}\left|A_{\tilde{c}}^{(j)}\left(x_{0}\right)-\left(-\tilde{\gamma}_{c}^{\mathrm{I}}\right)^{j} a_{c}^{\mathrm{I}} e^{-\tilde{\gamma}_{c}^{\mathrm{I}} x_{0}}\right| \leq C \delta e^{-\tilde{\gamma}_{c}^{\mathrm{I}} x_{0}} .
$$

From the equation of $A_{\tilde{c}}$, (4.32) and (4.36) (which implies for $x \geq x_{0},\left|\left(Q^{3} A_{c}\right)^{\prime}\right|+$ $\left.\left|G_{\tilde{c}}\right| \leq C \delta e^{-\frac{3}{2} x}\right)$, we have

$$
\forall x>x_{0}, \quad\left|A_{\tilde{c}}^{\prime \prime \prime}-A_{\tilde{c}}^{\prime}-\sqrt{\tilde{c}}(1-\tilde{c}) A_{\tilde{c}}\right| \leq C \delta e^{-\frac{3}{2} x} .
$$

Now, it follows from (4.38), (4.39) and standard ODE arguments (Duhamel formula) that

$$
\forall x>x_{0}, \quad\left|A_{\tilde{c}}(x)-a_{c}^{\mathrm{I}} e^{-\tilde{\gamma}_{c}^{\mathrm{I}} x}\right| \leq C \delta e^{-\tilde{\gamma}_{c}^{\mathrm{I}} x}
$$

and thus $\left|a_{c}^{\mathrm{I}}-a_{\tilde{c}}^{\mathrm{I}}\right| \leq C \delta$.

The construction of the approximate solution requires the introduction of solutions of other ODEs but no refined property of these solutions is needed. We state two lemmas similar to Lemma 4.2 (i)-(ii), whose proofs are direct consequences of Claim 2.

Lemma 4.5. Let $1<c<\frac{4}{3}$. There exists a unique solution $A_{c} \in H^{3}$ of

$$
\left(L A_{c}\right)^{\prime}+\sqrt{c}(c-1) A_{c}=G_{c}^{\prime} \text { where } G_{c}(x)=e^{\sqrt{c} x} Q^{3}(x),
$$

such that

$$
\left|A_{c}^{(k)}(x)\right| \lesssim e^{\gamma_{c}^{0} x} \quad \text { for } x<0, \quad\left|A_{c}^{(k)}(x)\right| \lesssim e^{-\gamma_{c}^{\mathrm{I}} x} \quad \text { for } x>0,
$$


where

$$
\begin{gathered}
\gamma_{c}^{0}=\sqrt{c}, \quad \gamma_{c}^{\mathrm{I}}=\frac{\sqrt{c}}{2}-\sqrt{1-\frac{3}{4} c}, \quad \gamma_{c}^{\mathrm{II}}=\frac{\sqrt{c}}{2}+\sqrt{1-\frac{3}{4} c}, \\
0<\gamma_{c}^{\mathrm{I}}<\gamma_{c}^{\mathrm{II}}<\gamma_{c}^{0}=\gamma_{c}^{\mathrm{I}}+\gamma_{c}^{\mathrm{II}}=\sqrt{c} .
\end{gathered}
$$

Moreover, there exist $a_{c}^{\mathrm{I}, \mathrm{II}}$ such that

$$
\left|(d / d x)^{k}\left(A_{c}(x)-a_{c}^{\mathrm{I}} e^{-\gamma_{c}^{\mathrm{I}} x}-a_{c}^{\mathrm{II}} e^{-\gamma_{c}^{\mathrm{II}} x}\right)\right| \lesssim e^{-\frac{3}{2}|x|} \quad \text { for } x>0 .
$$

It is easily checked that $\gamma_{c}^{0},-\gamma_{c}^{\mathrm{I}, \mathrm{II}}$ are the roots of $-\gamma^{3}+\gamma+\sqrt{c}(c-1)$.

Lemma 4.6. Let $\frac{3}{4} \leq c \leq \frac{4}{3}, c \neq 1$ and $\frac{3}{4} \leq c^{\prime}<1$. There exist unique solutions $A_{c, c^{\prime}}^{\mathrm{I}}, A_{c, c^{\prime}}^{\mathrm{II}} \in H^{3}$ of

$$
\left(L A_{c, c^{\prime}}^{\mathrm{I}, \mathrm{II}}\right)^{\prime}+\left(c^{\prime \frac{3}{2}} \sqrt{c}|1-c|+\sqrt{c^{\prime}} \gamma_{c}^{\mathrm{I} \mathrm{II}}\left(1-c^{\prime}\right)\right) A_{c, c^{\prime}}^{\mathrm{I}, \mathrm{II}}=\left(G_{c, c^{\prime}}^{\mathrm{I}, \mathrm{II}}\right)^{\prime},
$$

where

$$
G_{c, c^{\prime}}^{\mathrm{I}, \mathrm{II}}(x)=e^{-\sqrt{c^{\prime}} \gamma_{c}^{\mathrm{I}, \mathrm{II}} x} Q^{3}(x)
$$

Moreover,

$\left|\left(A_{c, c^{\prime}}^{\mathrm{I}, \mathrm{II}}\right)^{(k)}(x)\right| \lesssim e^{\left(\gamma_{c, c^{\prime}}+\gamma_{c}^{\mathrm{I}, \mathrm{II}} \sqrt{c^{\prime}}\right) x} \quad$ for $x<0, \quad\left|\left(A_{c, c^{\prime}}^{\mathrm{I}, \mathrm{II}}\right)^{(k)}(x)\right| \lesssim e^{-\gamma_{c, c^{\prime}} x} \quad$ for $x>0$,

where

$$
\gamma_{c, c^{\prime}}=\sqrt{1-\frac{3}{4}\left(\gamma_{c}^{\mathrm{I}, \mathrm{II}}\right)^{2} c^{\prime}}-\frac{\gamma_{c}^{\mathrm{I}, \mathrm{II}} \sqrt{c^{\prime}}}{2}
$$

Moreover,

$$
\gamma_{c, c^{\prime}} \geq 1-\gamma_{c}^{\mathrm{I}, \mathrm{II}} \sqrt{c^{\prime}}
$$

Note that

$$
c^{\prime \frac{3}{2}} \sqrt{c}|1-c|+\sqrt{c^{\prime}} \gamma_{c}^{\mathrm{I}, \mathrm{II}}\left(1-c^{\prime}\right)=\sqrt{c^{\prime}} \gamma_{c}^{\mathrm{I}, \mathrm{II}}\left(1-c^{\prime}\left(\gamma_{c}^{\mathrm{I}, \mathrm{II}}\right)^{2}\right) .
$$

One then easily checks that the three roots of $-\gamma^{3}+\gamma+c^{\prime \frac{3}{2}} \sqrt{c}|1-c|+\sqrt{c^{\prime}} \gamma_{c}^{\mathrm{I}, \mathrm{II}}\left(1-c^{\prime}\right)$ are

$$
\gamma_{c, c^{\prime}}+\gamma_{c}^{\mathrm{I}, \mathrm{II}} \sqrt{c^{\prime}},-\gamma_{c, c^{\prime}},-\sqrt{c^{\prime}} \gamma_{c}^{\mathrm{I}, \mathrm{II}}
$$

Equivalently, we can apply Lemma 4.2 with $c^{\prime}\left(\gamma_{c}^{\mathrm{I}, \mathrm{II}}\right)^{2}$ instead of $c$. Note that inequality (4.47) is a direct consequence of (3.10).

For future use in the construction of the approximate solution, we now define rescaled versions of $A_{c}$ and $A_{c, c^{\prime}}$ and we gather useful information about these functions in the next lemma. Let

$$
\begin{aligned}
& A_{j, k}(x)=c_{j}^{\frac{1}{3}} A_{c_{k} / c_{j}}\left(c_{j}^{\frac{1}{2}} x\right), \quad \gamma_{j, k}^{0}=c_{j}^{\frac{1}{2}} \gamma_{c_{k} / c_{j}}^{0}, \quad \gamma_{j, k}^{\mathrm{I}, \mathrm{II}}=c_{j}^{\frac{1}{2}} \gamma_{c_{k} / c_{j}}^{\mathrm{I}, \mathrm{II}}, \quad a_{j, k}^{\mathrm{I}, \mathrm{II}}=c_{j}^{\frac{1}{3}} a_{c_{k} / c_{j}}^{\mathrm{I}, \mathrm{II}}, \\
& A_{j, k, l}^{\mathrm{I}, \mathrm{II}}(x)=c_{l}^{\frac{1}{3}} A_{c_{k} / c_{j}, c_{j} / c_{l}}^{\mathrm{I}, \mathrm{II}}\left(c_{l}^{\frac{1}{2}} x\right) .
\end{aligned}
$$

Lemma 4.7. Assume

$$
\frac{3}{4}<\frac{c_{k}}{c_{j}}<\frac{4}{3}
$$


(i) For $j<k, A_{j, k}$ satisfies

$$
\begin{gathered}
\left(L_{c_{j}} A_{j, k}\right)^{\prime}+\sqrt{c_{k}}\left(c_{j}-c_{k}\right) A_{j, k}=\left(e^{-\sqrt{c_{k}} x} Q_{c_{j}}^{3}\right)^{\prime} . \\
\left|A_{j, k}(x)\right| \lesssim e^{\gamma_{j, k}^{0}} \quad \text { for } x<0, \\
\left|d^{k} / d x^{k}\left(A_{j, k}(x)-a_{j, k}^{\mathrm{I}} e^{-\gamma_{j, k}^{\mathrm{I}} x}-a_{j, k}^{\mathrm{II}} e^{-\gamma_{j, k}^{\mathrm{II}} x}\right)\right| \lesssim e^{-\frac{9}{8}|x|} . \\
\gamma_{j, k}^{0}=\sqrt{c_{j}-\frac{3}{4} c_{k}}+\frac{1}{2} \sqrt{c_{k}}, \quad \gamma_{j, k}^{\mathrm{I}}=\sqrt{c_{j}-\frac{3}{4} c_{k}}-\frac{1}{2} \sqrt{c_{k}}, \quad \gamma_{j, k}^{\mathrm{II}}=\sqrt{c_{k}} . \\
\text { Moreover, } \\
a_{j, k}^{\mathrm{I}} \neq 0 \text { and its sign does not depend on } j \text { and } k .
\end{gathered}
$$

(ii) For $j>k, A_{j, k}$ satisfies

$$
\begin{gathered}
\left(L_{c_{j}} A_{j, k}\right)^{\prime}+\sqrt{c_{k}}\left(c_{k}-c_{j}\right) A_{j, k}=\left(e^{\sqrt{c_{k}} x} Q_{c_{j}}^{3}\right)^{\prime} . \\
\left|A_{j, k}(x)\right| \lesssim e^{\gamma_{j, k}^{0}} \quad \text { for } x<0, \\
\left|d^{k} / d x^{k}\left(A_{j, k}(x)-a_{j, k}^{\mathrm{I}} e^{-\gamma_{j, k}^{\mathrm{I}} x}-a_{j, k}^{\mathrm{II}} e^{-\gamma_{j, k}^{\mathrm{II}} x}\right)\right| \lesssim e^{-\frac{9}{8}|x|} . \\
\gamma_{j, k}^{0}=\sqrt{c_{k}}, \quad \gamma_{j, k}^{\mathrm{I}}=\frac{1}{2} \sqrt{c_{k}}-\sqrt{c_{j}-\frac{3}{4} c_{k}}, \quad \gamma_{j, k}^{\mathrm{II}}=\frac{1}{2} \sqrt{c_{k}}+\sqrt{c_{j}-\frac{3}{4} c_{k} .}
\end{gathered}
$$

Moreover,

$$
j<k \Rightarrow \gamma_{j, k}^{\mathrm{I}}<\gamma_{k, j}^{\mathrm{I}} .
$$

(iii) For $j \neq k, l<j, A_{j, k, l}^{\mathrm{I}}$ and $A_{j, k, l}^{\mathrm{II}}$ satisfy

$$
\begin{gathered}
\left(L_{c_{l}} A_{j, k, l}^{\mathrm{I}, \mathrm{II}}\right)^{\prime}+\left(\sqrt{c_{k}}\left|c_{j}-c_{k}\right|+\gamma_{j, k}^{\mathrm{I}, \mathrm{II}}\left(c_{l}-c_{j}\right)\right) A_{j, k, l}^{\mathrm{I}, \mathrm{II}}=\left(e^{-\gamma_{j, k}^{\mathrm{I}, \mathrm{II}} x} Q_{c_{l}}^{3}\right)^{\prime} . \\
\left|A_{j, k, l}^{\mathrm{I}, \mathrm{II}}(x)\right| \lesssim e^{\sqrt{c_{l}} x} \quad \text { for } x<0, \quad\left|A_{j, k, l}^{\mathrm{I}, \mathrm{II}}(x)\right| \lesssim e^{-\left(\sqrt{c_{l}}-\gamma_{j, k}^{\mathrm{I}, \mathrm{II}}\right) x}, \quad \text { for } x>0 .
\end{gathered}
$$

Proof of (4.52). This property follows directly from Lemma 4.2 (iv).

Proof of (4.57). It is equivalent to prove

$$
\sqrt{c_{j}-\frac{3}{4} c_{k}}-\frac{1}{2} \sqrt{c_{j}}<\frac{1}{2} \sqrt{c_{k}}-\sqrt{c_{k}-\frac{3}{4} c_{j}}
$$

which is clear since from $c_{j}>c_{k}$, we have

$$
\sqrt{c_{j}-\frac{3}{4} c_{k}}-\frac{1}{2} \sqrt{c_{j}}=\frac{\frac{3}{4}\left(c_{j}-c_{k}\right)}{\sqrt{c_{j}-\frac{3}{4} c_{k}}+\frac{1}{2} \sqrt{c_{j}}}<\frac{\frac{3}{4}\left(c_{j}-c_{k}\right)}{\sqrt{c_{k}-\frac{3}{4} c_{j}}+\frac{1}{2} \sqrt{c_{k}}}=\frac{1}{2} \sqrt{c_{k}}-\sqrt{c_{k}-\frac{3}{4} c_{j}} .
$$

Proof of (4.59). It is a consequence of (4.47). Note that

$$
\gamma_{j, k}^{\mathrm{II}}<\sqrt{c_{l}} .
$$

Indeed, if $k \leq l$, then $\gamma_{j, k}^{\mathrm{II}}<\gamma_{l, k}^{\mathrm{II}} \leq \sqrt{c_{l}}$. If $j<k<l$, then $\gamma_{j, k}^{\mathrm{II}}<\sqrt{c_{k}}<\sqrt{c_{l}}$. If $k<j<l$, then $\gamma_{j, k}^{\mathrm{II}}=\sqrt{c_{k}}<\sqrt{c_{l}}$. 
4.2. Construction of the approximate solution. In this subsection, we use the functions defined in Lemma 4.7 to construct an approximate solution. In this construction, we denote by $E_{i}$ (for $\left.i=1, \ldots, 5\right)$ error terms of size $e^{-2 \sigma_{0} t}$. See Claim 6.

4.2.1. Two soliton interactions. Inserting

$$
R=\sum_{j} R_{j}, \quad R_{j}(t, x)=Q\left(x-y_{j}(t)\right), \quad y_{j}(t)=c_{j} t+\Delta_{j}
$$

as a first approximation into the quartic $(\mathrm{gKdV})$ equation, since $\partial_{t} R_{j}+\partial_{x}\left(\partial_{x}^{2} R_{j}+\right.$ $\left.R_{j}^{4}\right)=0$, we find

$$
\partial_{t} R+\partial_{x}\left(\partial_{x}^{2} R+R^{4}\right)=\partial_{x}\left(R^{4}-\sum_{j} R_{j}^{4}\right)=\partial_{x}\left(4 \sum_{j \neq k} R_{k} R_{j}^{3}+E_{1}\right)
$$

where, for some $n_{1}, n_{2}, n_{3}$,

$$
E_{1}=n_{1} \sum_{j_{1} \neq j_{2}} R_{j_{1}}^{2} R_{j_{2}}^{2}+n_{2} \sum_{j_{k} \neq j_{l}} R_{j_{1}}^{2} R_{j_{2}} R_{j_{3}}+n_{3} \sum_{j_{k} \neq j_{l}} R_{j_{1}} R_{j_{2}} R_{j_{3}} R_{j_{4}} .
$$

The error term $E_{1}$ is controlled in Claim 6

The term $R_{k} R_{j}^{3}$ cannot be considered as an error term. In fact, such term will contribute to the lower bound on the approximate solution which is the key point of the proof of Theorem 1.1. For this term, it is convenient to decouple the variables of $R_{k}$ and $R_{j}$ by approximating $R_{k}$ by its asymptotic expansion around $R_{j}$ (see proof of Claim 6 for more details). Since

$$
4 R_{k}(t, x) \underset{x \sim y_{j}}{\approx} 4(10)^{\frac{1}{3}} c_{k}^{\frac{1}{3}} e^{-\sqrt{c_{k}}\left|x-y_{k}\right|}=z_{j, k} e^{-\iota_{j, k} \sqrt{c_{k}}\left(x-y_{j}\right)},
$$

where $\iota_{j, k}=\operatorname{sgn}(k-j)$ and

$$
z_{j, k}(t)=4(10)^{\frac{1}{3}} c_{k}^{\frac{1}{3}} e^{-\iota_{j, k} \sqrt{c_{k}}\left(\Delta_{j}-\Delta_{k}\right)} e^{-\sqrt{c_{k}}\left|c_{j}-c_{k}\right| t},
$$

we rewrite the second member of the equation of $R$ as follows

$$
\partial_{t} R+\partial_{x}\left(\partial_{x}^{2} R+R^{4}\right)=\partial_{x}\left(\sum_{j \neq k} z_{j, k} e^{-\iota_{j, k} \sqrt{c_{k}}\left(x-y_{j}\right)} R_{j}^{3}\right)+\partial_{x}\left(E_{1}+E_{2}\right),
$$

where $E_{2}$, to be controlled in Claim 6, is the error term generated by this approximation

$$
E_{2}=\sum_{j \neq k} R_{j}^{3}\left(4 R_{k}-z_{j, k} e^{-\iota_{j, k} \sqrt{c_{k}}\left(x-y_{j}\right)}\right) .
$$

4.2.2. First correction and three soliton interactions. We define an improved version of the approximate solution to cancel the main terms in the right-hand side of (4.61). Let

$$
\bar{V}:=R+Z, \quad Z=\sum_{\substack{j, k=1, \ldots, N \\ j \neq k}} Z_{j, k}
$$

where

$$
Z_{j, k}(t, x)=z_{j, k}(t) A_{j, k}\left(x-y_{j}(t)\right), \quad j \neq k .
$$


By the equation of $A_{j, k}$ in (4.48), (4.53) we get:

$$
\begin{aligned}
\partial_{t} Z_{j, k}+\partial_{x}\left(\partial_{x}^{2} Z_{j, k}+4 R_{j}^{3} Z_{j, k}\right) & =z_{j, k}\left(-\sqrt{c_{k}}\left|c_{j}-c_{k}\right| A_{j, k}-\left(L_{c_{j}} A_{j, k}\right)^{\prime}\right)\left(x-y_{j}\right) \\
& =-\partial_{x}\left(z_{j, k} e^{-\iota_{j, k} \sqrt{c_{k}}\left(x-y_{j}\right)} R_{j}^{3}\right) .
\end{aligned}
$$

Therefore, using (4.61),

$$
\begin{aligned}
\partial_{t} \bar{V}+\partial_{x}\left(\partial_{x}^{2} \bar{V}+\bar{V}^{4}\right) & =\partial_{t} R+\partial_{x}\left(\partial_{x}^{2} R+R^{4}\right)-\partial_{x}\left(\sum_{j \neq k} z_{j, k} e^{-\iota_{j, k} \sqrt{c_{k}}\left(x-y_{j}\right)} R_{j}^{3}\right) \\
& +\partial_{x}\left((R+Z)^{4}-R^{4}-4 \sum_{j \neq k} R_{j}^{3} Z_{j, k}\right) \\
& =\partial_{x}\left((R+Z)^{4}-R^{4}-4 \sum_{j \neq k} R_{j}^{3} Z_{j, k}\right)+\partial_{x}\left(E_{1}+E_{2}\right) \\
& =\partial_{x}\left(4 \sum_{j \neq k, j>l} R_{l}^{3} Z_{j, k}\right)+\partial_{x}\left(E_{1}+E_{2}+E_{3}\right) .
\end{aligned}
$$

where

$$
E_{3}=4 R^{3} Z-4\left(\sum_{j \neq k} R_{j}^{3} Z_{j, k}+\sum_{j \neq k, j>l} R_{l}^{3} Z_{j, k}\right)+6 R^{2} Z^{2}+4 R Z^{3}+Z^{4} .
$$

In the right-hand side of (4.63), the term $\partial_{x}\left(\sum_{j \neq k, j>l} R_{l}^{3} Z_{j, k}\right)$ is not an error term in the sense that it is not of size $e^{-2 \sigma_{0} t}$. We use the asymptotic expansion of $A_{j, k}$ in (4.50) and (4.55) (depending on $j<k$ or $k<j$ ) to replace $Z_{j, k}$ in this term by its asymptotic expansion near $y_{l}$ (since $j>l$, we have $c_{l}>c_{j}$ and $y_{l} \gg y_{j}$ for $t$ large)

$$
\begin{aligned}
4 Z_{j, k}(t, x) \underset{x \sim y_{l}}{\approx} 4 z_{j, k} a_{j, k}^{\mathrm{I}} e^{-\gamma_{j, k}^{\mathrm{I}}\left(x-y_{j}\right)}+4 z_{j, k} a_{j, k}^{\mathrm{II}} e^{-\gamma_{j, k}^{\mathrm{II}}\left(x-y_{j}\right)} \\
\underset{x \sim y_{l}}{\approx} z_{j, k}^{\mathrm{I}} e^{-\gamma_{j, k}^{\mathrm{I}}\left(x-y_{l}\right)}+z_{j, k}^{\mathrm{II}} e^{-\gamma_{j, k}^{\mathrm{II}}\left(x-y_{l}\right)}
\end{aligned}
$$

where

$$
z_{j, k, l}^{\mathrm{I}, \mathrm{II}}(t)=4 z_{j, k}(t) a_{j, k}^{\mathrm{I}, \mathrm{II}} e^{-\gamma_{j, k}^{\mathrm{I}, \mathrm{II}}\left(\Delta_{l}-\Delta_{j}\right)} e^{-\gamma_{j, k}^{\mathrm{I}, \mathrm{II}}\left(c_{l}-c_{j}\right) t} .
$$

We obtain

$$
\begin{aligned}
\partial_{t} \bar{V}+\partial_{x}\left(\partial_{x}^{2} \bar{V}+\bar{V}^{4}\right) & =\partial_{x}\left(\sum_{j \neq k, j>l} z_{j, k}^{\mathrm{I}} e^{-\gamma_{j, k}^{\mathrm{I}}\left(x-y_{l}\right)} R_{l}^{3}+z_{j, k}^{\mathrm{II}} e^{-\gamma_{j, k}^{\mathrm{II}}\left(x-y_{l}\right)} R_{l}^{3}\right) \\
& +\partial_{x}\left(\sum_{i=1}^{4} E_{i}\right)
\end{aligned}
$$

where

$$
E_{4}=\sum_{j \neq k, j>l}\left(4 Z_{j, k}-z_{j, k}^{\mathrm{I}} e^{-\gamma_{j, k}^{\mathrm{I}}\left(x-y_{l}\right)}-z_{j, k}^{\mathrm{II}} e^{-\gamma_{j, k}^{\mathrm{II}}\left(x-y_{l}\right)}\right) R_{l}^{3}
$$


4.2.3. Second correction and final approximate solution. We refine the above approximate solution $\bar{V}$ to remove the main terms in the right-hand side of (4.64). We now define $V$ the final version of approximate solution

$$
\begin{gathered}
V:=\bar{V}+W=R+Z+W, \\
W=\sum_{j=2}^{N} \sum_{\substack{k=1 \\
k \neq j}}^{N} \sum_{l=1}^{j-1}\left(Z_{j, k, l}^{\mathrm{I}}+Z_{j, k, l}^{\mathrm{II}}\right),
\end{gathered}
$$

where

$$
Z_{j, k, l}^{\mathrm{I}, \mathrm{II}}(t, x)=z_{j, k, l}^{\mathrm{I}, \mathrm{II}}(t) A_{j, k, l}^{\mathrm{I}, \mathrm{II}}\left(x-y_{l}(t)\right), \quad j \neq k, 1 \leq l<j .
$$

First, we observe that for such $V$, (4.3) follows directly from the definition of $\sigma_{0}$ and $z_{j, k}, z_{j, k}^{\mathrm{I}, \mathrm{II}}$,

$$
\|V(t)-R(t)\|_{H^{3}} \leq\|Z(t)\|_{H^{3}}+\|W(t)\|_{H^{3}} \leq C \sum_{j \neq k} z_{j, k}(t) \leq C e^{-\sigma_{0} t} .
$$

Now, we prove that (4.4) holds for $V$. From the equation of $A_{j, k, l}^{\mathrm{I}, \mathrm{II}}$ in (4.58), we have

$$
\begin{aligned}
& \partial_{t} Z_{j, k, l}^{\mathrm{I}, \mathrm{II}}+\partial_{x}\left(\partial_{x}^{2} Z_{j, k, l}^{\mathrm{I}, \mathrm{II}}+4 R_{l}^{3} Z_{j, k, l}^{\mathrm{I}, \mathrm{II}}\right) \\
& =z_{j, k, l}^{\mathrm{I}, \mathrm{II}}\left(-\left(\sqrt{c_{k}}\left|c_{j}-c_{k}\right|+\gamma_{j, k}^{\mathrm{I}, \mathrm{II}}\left(c_{l}-c_{j}\right)\right) A_{j, k, l}^{\mathrm{I}, \mathrm{II}}-\left(L_{c_{l}} A_{j, k, l}^{\mathrm{I}, \mathrm{II}}\right)^{\prime}\right)\left(x-y_{l}\right) \\
& =-\partial_{x}\left(z_{j, k}^{\mathrm{I}, \mathrm{II}} e^{-\gamma_{j, k}^{\mathrm{I}, \mathrm{II}}\left(x-y_{l}\right)} R_{l}^{3}\right) .
\end{aligned}
$$

Therefore, using (4.64), we get

$$
\begin{aligned}
\partial_{t} V+\partial_{x}\left(\partial_{x}^{2} V+V^{4}\right) & =\partial_{t} \bar{V}+\partial_{x}\left(\partial_{x}^{2} \bar{V}+\bar{V}^{4}\right) \\
& -\partial_{x}\left(\sum_{j \neq k, j>l} z_{j, k}^{\mathrm{I}} e^{-\gamma_{j, k}^{\mathrm{I}}\left(x-y_{l}\right)} R_{l}^{3}+z_{j, k}^{\mathrm{II}} e^{-\gamma_{j, k}^{\mathrm{II}}\left(x-y_{l}\right)} R_{l}^{3}\right) \\
& +\partial_{x}\left(V^{4}-\bar{V}^{4}-4 \sum_{j \neq k, j>l} R_{l}^{3}\left(Z_{j, k, l}^{\mathrm{I}}+Z_{j, k, l}^{\mathrm{II}}\right)\right) \\
& =\partial_{x}\left(\sum_{i=1}^{5} E_{i}\right),
\end{aligned}
$$

where

$$
E_{5}=(R+Z+W)^{4}-(R+Z)^{4}-4 \sum_{j \neq k, j>l} R_{l}^{3}\left(Z_{j, k, l}^{\mathrm{I}}+Z_{j, k, l}^{\mathrm{II}}\right) .
$$

The following claim (see proof in Appendix C) completes the proof of (4.4).

Claim 6. For $i=1, \ldots, 5$,

$$
\left\|\partial_{x} E_{i}(t)\right\|_{H^{3}} \leq C e^{-2 \sigma_{0} t} .
$$


4.3. Asymptotics of the approximate solution. Proof of (4.5). First, it is clear that

$$
0<R_{j}(t, x)<C e^{-\sqrt{c_{j}}\left(x-c_{j} t\right)},
$$

so that

$$
R_{j}\left(t, x_{0}(t)\right) \lesssim e^{-\sqrt{c_{j}}\left(x_{0}(t)-c_{j} t\right)} .
$$

By (3.6) and (3.8), we have $\frac{\sigma_{0}}{\gamma_{0}}>c_{j_{0}+1}$ and $\frac{\sigma_{0}}{\gamma_{0}}+c_{j_{0}}>5 \sigma_{0}+\frac{16}{25}$. Therefore,

$$
\begin{aligned}
\sqrt{c_{j}}\left(x_{0}(t)-c_{j} t\right) & \geq \frac{\sqrt{3}}{2}\left(\frac{4}{5 \sqrt{3}}\left(5 \sigma_{0}+\frac{16}{25}\right)+\frac{3}{2}\left(1-\frac{4}{5 \sqrt{3}}\right)-1\right) t-K_{0} \\
& \geq\left(2 \sigma_{0}+\frac{\sqrt{3}}{2}\left(\frac{64}{125 \sqrt{3}}+\frac{1}{2}-\frac{6}{5 \sqrt{3}}\right)\right) t-K_{0} \\
& \geq 2 \sigma_{0} t+\frac{1}{10} t-K_{0} .
\end{aligned}
$$

It follows that, for $t>0$,

$$
0<R\left(t, x_{0}(t)\right)<C e^{-\frac{1}{10} t} e^{-2 \sigma_{0} t} e^{K_{0}} .
$$

Second, for $1 \leq j<k \leq N$, we have by (4.50)-(4.52), for all $x-c_{j} t \gg 1$,

$$
\left|Z_{j, k}(t, x)\right| \geq \kappa e^{-\sqrt{c_{k}}\left(c_{j}-c_{k}\right) t} e^{-\gamma_{j, k}^{\mathrm{I}}\left(x-c_{j} t\right)} .
$$

Since all $Z_{j, k}$ for $j<k$ have the same sign at $+\infty$ (see (4.52)), their contributions are added and we obtain, for all $x-t \gg 1$,

$$
\left|\sum_{j<k} Z_{j, k}(t, x)\right| \geq \kappa \sum_{j<k} e^{-\sqrt{c_{k}}\left(c_{j}-c_{k}\right) t} e^{-\gamma_{j, k}^{\mathrm{I}}\left(x-c_{j} t\right)} .
$$

Moreover, using (4.57), for $x-c_{j} t \gg 1$,

$$
\begin{aligned}
\left|Z_{k, j}(t, x)\right| & \leq C e^{-\sqrt{c_{j}}\left(c_{j}-c_{k}\right) t} e^{-\gamma_{k, j}^{\mathrm{I}}\left(x-c_{k} t\right)} \\
& \leq C e^{-\left(\sqrt{c_{j}}-\sqrt{c_{k}}\right)\left(c_{j}-c_{k}\right) t} e^{-\sqrt{c_{k}}\left(c_{j}-c_{k}\right) t} e^{-\gamma_{j, k}^{\mathrm{I}}\left(x-c_{j} t\right)} \\
& \leq C e^{-\left(\sqrt{c_{j}}-\sqrt{c_{k}}\right)\left(c_{j}-c_{k}\right) t}\left|Z_{j, k}(t, x)\right| .
\end{aligned}
$$

Thus, for $t$ large enough, and $x-t \gg 1$,

$$
|Z(t, x)| \geq \frac{\kappa}{2} \sum_{j<k} e^{-\sqrt{c_{k}}\left(c_{j}-c_{k}\right) t} e^{-\gamma_{j, k}^{\mathrm{I}}\left(x-c_{j} t\right)} .
$$

and

$$
\left|Z\left(t, x_{0}(t)\right)\right| \geq \frac{\kappa}{2} \sum_{j<k} e^{-\sqrt{c_{k}}\left(c_{j}-c_{k}\right) t} e^{-\gamma_{j, k}^{\mathrm{I}}\left(x_{0}(t)-c_{j} t\right)} \geq \frac{\kappa}{2} e^{\gamma_{0} K_{0}} e^{2 \sigma_{0} t} .
$$

Third, to control $W_{j, k, l}$, we use (4.59). For $j \neq k, j>l, x-t>0$, we have

$$
\left|Z_{j, k, l}^{\mathrm{I}}(t, x)\right| \leq C e^{-\sqrt{c_{k}}\left|c_{j}-c_{k}\right| t} e^{-\gamma_{j, k}^{\mathrm{I}}\left(c_{l}-c_{j}\right) t} e^{-\left(\sqrt{c_{l}}-\gamma_{j, k}^{\mathrm{I}}\right)\left(x-c_{l} t\right)} .
$$

In the case where $\left(c_{l}-c_{j}\right) t>\frac{3}{4}\left(x-c_{l} t\right)$, we obtain

$$
\left|Z_{j, k, l}^{\mathrm{I}}(t, x)\right| \leq C e^{-\sqrt{c_{k}}\left|c_{j}-c_{k}\right| t} R_{l}^{\frac{3}{4}}(t, x) \leq e^{-4 \sigma_{0} t}+R_{l}(t, x) .
$$


In the case where $\left(c_{l}-c_{j}\right) t<\frac{3}{4}\left(x-c_{l} t\right)$, we obtain

$$
\left|Z_{j, k, l}^{\mathrm{I}}(t, x)\right| \leq C e^{-\sqrt{c_{k}}\left|c_{j}-c_{k}\right| t} e^{-\sqrt{c_{l}}\left(c_{l}-c_{j}\right) t} e^{-\frac{1}{3}\left(\sqrt{c_{l}}-\gamma_{j, k}^{\mathrm{I}}\right)\left(c_{l}-c_{j}\right) t} .
$$

In particular, for $t$ large enough (depending on $K_{0}$ ), we obtain

$$
\mid R\left(t, x_{0}(t)|+| W\left(t, x_{0}(t)\right)\left|\leq \frac{1}{10}\right| Z\left(t, x_{0}(t)\right) \mid\right.
$$

and so

$$
\left|(R+Z+W)\left(t, x_{0}(t)\right)\right| \geq \frac{\kappa}{3} e^{\gamma_{0} K_{0}} e^{-2 \sigma_{0} t}
$$

\section{Lower bound for outgoing multi-soliton for $t \gg 1$}

In this section, we estimate the distance between the solution $u(t)$ of (1.1) satisfying (1.11) and the approximate solution $V(t)$ constructed in Section 4 . The strategy of the proof follows closely Proposition 6 in [11] (see also [19]). From (4.5) and this estimate, we deduce a lower bound on $\left|u\left(t, x_{0}(t)\right)\right|$ for large time.

Proposition 5.1. Assume (1.9) and (1.10).

(i) Comparison with the approximate solution. There exists $C>0$ such that for all $t>0$,

$$
\|u(t)-V(t)\|_{H^{1}} \leq C e^{-2 \sigma_{0} t}
$$

(ii) Lower bound. There exist $\kappa_{1}>0$ and $t_{1}\left(K_{0}\right)>0$ such that for $K_{0}>0$ large enough, for all $t>t_{1}\left(K_{0}\right)$,

$$
\left|u\left(t, x_{0}(t)\right)\right| \geq \kappa_{1} e^{\gamma_{0} K_{0}} e^{-2 \sigma_{0} t},
$$

where $x_{0}(t)=\left(\frac{\sigma_{0}}{\gamma_{0}}+c_{j_{0}}\right) t-K_{0}$.

Proof. Proof of (i). The proof is similar to the one of Proposition 6 in [11], so we only sketch it. Let

$$
w(t)=u(t)-V(t)
$$

Let

$$
R(t, x)=\sum_{j=1}^{N} R_{j}(t, x), \quad R_{j}(t, x)=Q\left(x-c_{j} t-\Delta_{j}\right) .
$$

On the one hand, by Theorem 1 in [1], $u(t) \in H^{3}$, and there exists $\bar{\sigma}>0$ such that

$$
\|u(t)-R(t)\|_{H^{3}} \leq C e^{-\bar{\sigma} t} .
$$

On the other hand, by (4.3),

$$
\|V(t)-R(t)\|_{H^{3}} \leq C e^{-\sigma_{0} t}
$$

Thus, for some $\sigma>0$,

$$
\|w(t)\|_{H^{3}} \leq C e^{-\sigma t} .
$$

Next, note that $w$ satisfies the following equation

$$
w_{t}+\left(w_{x x}+(V+w)^{4}-V^{4}\right)_{x}+E(V)=0,
$$


where $E(V)=\partial_{t} V+\partial_{x}\left(\partial_{x}^{2} V+V^{4}\right)$. Define

$\mathcal{F}(t)=\int\left\{\left(w_{x}^{2}-\frac{2}{5}\left((V+w)^{5}-V^{5}-5 w V^{4}\right)\right)(t) f(t)+w^{2}(t)\right\}$

where $f(t, x)=\frac{1}{c_{N}}-\sum_{j=1}^{N-1}\left(\frac{1}{c_{j+1}}-\frac{1}{c_{j}}\right) \phi\left(\sqrt{\sigma}\left(x-\frac{c_{j+1}+c_{j}}{2} t-\frac{\Delta_{j+1}+\Delta_{j}}{2}\right)\right)$

and

$$
\sigma=\frac{1}{4} \min \left(c_{1}-c_{2}, \ldots, c_{N-1}-c_{N}, c_{N}\right)
$$

We claim

Claim 7 (Energy estimate). There exist $C, \sigma_{1}>0$ such that, for $t \geq 0$,

$$
\mathcal{F}(t) \leq C e^{-\sigma_{1} t} \sup _{t^{\prime} \geq t}\left\|w\left(t^{\prime}\right)\right\|_{H^{1}}^{2}+C e^{-2 \sigma_{0} t} \sup _{t^{\prime} \geq t}\left\|w\left(t^{\prime}\right)\right\|_{H^{1}}
$$

Sketch of the proof of Claim [7. The proof is similar to the one of Lemma 4 [11]. The only difference is the presence of the error term $E(V)$ in (5.5), which generates the second term in the right-hand side of (5.6).

The proof relies on the following estimate of the time derivative of $\mathcal{F}$ : for some $\sigma_{1}>0$,

$$
\frac{d \mathcal{F}}{d t}(t) \geq-C e^{-\sigma_{1} t}\|w\|_{H^{1}}^{2}-C e^{-2 \sigma_{0} t}\|w\|_{L^{2}} .
$$

Integrating (5.7) on $\left[t,+\infty\right.$ ), since $\lim _{t \rightarrow \infty} \mathcal{F}(t)=0$ (by (5.4) ), Claim 7 is proved.

The proof of (5.7) is omitted (see [11]). We only recall that a key step of the proof is the following property of $V$

$$
\left\|V_{t} f+V_{x}\right\|_{L^{\infty}} \leq C e^{-\sigma_{1} t}
$$

for some $\sigma_{1}>0$, easily proved using (5.3) and (4.4).

Next, we claim without proof the following direct consequence of the equations of $w$ and $Q_{c_{j}}$.

Claim 8 (Control of the scaling directions).

$$
\sum_{j=1}^{N}\left|\int w(t) R_{j}(t)\right| \leq C e^{-\sigma_{1} t} \sup _{t^{\prime} \geq t}\left\|w\left(t^{\prime}\right)\right\|_{H^{1}}+C e^{-2 \sigma_{0} t} .
$$

We now control the translation directions and conclude the proof. Let

$$
\begin{gathered}
\tilde{w}(t)=w(t)+\sum_{j=1}^{N} a_{j}(t)\left(R_{j}\right)_{x}(t), \quad a_{j}(t)=-\frac{\int w(t)\left(R_{j}\right)_{x}(t)}{\int\left(R_{j}\right)_{x}^{2}(t)}, \quad \int \tilde{w}(t)\left(R_{j}\right)_{x}(t)=0, \\
C_{1}\|w(t)\|_{H^{1}}^{2} \leq\|\tilde{w}(t)\|_{H^{1}}^{2}+\sum_{j=1}^{N}\left|a_{j}(t)\right|^{2} \leq C_{2}\|w(t)\|_{H^{1}}^{2}
\end{gathered}
$$

We claim the following result, based on the equations of $\left(R_{j}\right)_{x}$, Claims 7 and 8 , as well as a coercivity property of $\mathcal{F}$ up to scaling and translation. 
Claim 9. For $t>0$, for some $\sigma_{1}>0$,

$$
\|\tilde{w}(t)\|_{H^{1}}^{2}+\sum_{j=1}^{N}\left|a_{j}(t)\right|^{2} \leq C e^{-\sigma_{1} t} \sup _{t^{\prime} \geq t}\left\|w\left(t^{\prime}\right)\right\|_{H^{1}}^{2}+C e^{-4 \sigma_{0} t} .
$$

From (5.11) and (5.10), we obtain

$$
\|w(t)\|_{H^{1}}^{2} \leq C e^{-\sigma_{1} t} \sup _{t^{\prime} \geq t}\left\|w\left(t^{\prime}\right)\right\|_{H^{1}}^{2}+C e^{-4 \sigma_{0} t},
$$

and thus, for $t$ large enough,

$$
\frac{1}{2}\|w(t)\|_{H^{1}} \leq C e^{-2 \sigma_{0} t}
$$

which completes the proof of part (i) of Proposition 5.1.

Proof of (ii). Lower bound. From (4.5) and (5.1), for $t>t_{1}\left(K_{0}\right)$,

$$
\begin{aligned}
\left|u\left(t, x_{0}(t)\right)\right| & \geq\left|V\left(t, x_{0}(t)\right)\right|-\left|u\left(t, x_{0}(t)\right)-V\left(t, x_{0}(t)\right)\right| \\
& \geq\left|V\left(t, x_{0}(t)\right)\right|-\|u(t)-V(t)\|_{H^{1}} \\
& \geq \kappa e^{\gamma_{0} K_{0}} e^{-2 \sigma_{0} t}-C e^{-2 \sigma_{0} t} \geq \frac{\kappa}{2} e^{\gamma_{0} K_{0}} e^{-2 \sigma_{0} t},
\end{aligned}
$$

for $K_{0}$ large enough.

\section{Appendix A. Proof of Lemma 3.1 and Claim 2.1}

A.1. Proof of Lemma 3.1. For $x_{0}>0, t \in\left[t_{1}, t_{2}\right]$, set the following energy and mass Liapunov functional:

$$
\begin{aligned}
& J_{x_{0}}(t)=\int\left(u_{x}^{2}+u^{2}-\frac{2}{5} u^{5}\right)(t) \psi(t) d x \\
& \text { where } \psi(t, x)=\phi\left(\sqrt{\sigma}\left(x-c_{0} t-\left(c_{0}-\sigma^{\prime}\right)\left(t_{2}-t\right)-x_{0}\right)\right)
\end{aligned}
$$

Estimate (3.3) is based on the control of the variation of $J_{x_{0}}$ on $\left[t_{1}, t_{2}\right]$. We claim

$$
\frac{d}{d t} J_{x_{0}}(t) \leq C e^{-\sqrt{\sigma}\left(c_{0}-\sigma^{\prime}\right)\left(t_{2}-t\right)} e^{-\sqrt{\sigma} x_{0}} .
$$

Indeed, we have by direct computations (see e.g. Appendix C in [14]),

$$
\begin{aligned}
\frac{d}{d t} J_{x_{0}}(t) & =\int\left(-\left(u_{x x}+u^{4}\right)^{2}-2 u_{x x}^{2}-3 u_{x}^{2}+8 u_{x}^{2} u^{3}+\frac{8}{5} u^{5}\right) \psi_{x} \\
& -\sigma^{\prime} \int\left(u_{x}^{2}+u^{2}-\frac{2}{5} u^{5}\right) \psi_{x}+\int\left(u_{x}^{2}+u^{2}\right) \psi_{x x x} .
\end{aligned}
$$

Thus, using (3.1),

$$
\begin{aligned}
\frac{d}{d t} J_{x_{0}}(t) & \leq \int\left(-\left(u_{x x}+u^{4}\right)^{2}-2 u_{x x}^{2}-3 u_{x}^{2}+8 u_{x}^{2}|u|^{3}+\frac{2}{5}\left(4+C_{0}\right)|u|^{5}\right) \psi_{x} \\
& -\left(\sigma^{\prime}-\sigma\right) \int\left(u_{x}^{2}+u^{2}\right) \psi_{x} .
\end{aligned}
$$


Note first that $\|u\|_{L^{\infty}\left(x>c_{0} t+R\right)}^{2} \leq\left\|u_{x}\right\|_{L^{2}\left(x>c_{0} t+R\right)}\|u\|_{L^{2}\left(x>c_{0} t+R\right)} \leq C_{0} \alpha_{0}$. Next, observe that

$$
\begin{aligned}
\int u_{x}^{2}|u|^{3} \psi_{x} & \leq \int_{x<c_{0} t+R} u_{x}^{2}|u|^{3} \psi_{x}+\int_{x>c_{0} t+R} u_{x}^{2}|u|^{3} \psi_{x} \\
& \leq C \phi^{\prime}\left(\sqrt{\sigma}\left(R-\left(c_{0}-\sigma^{\prime}\right)\left(t_{2}-t\right)-x_{0}\right)\right)+\|u\|_{L^{\infty}\left(x>c_{0} t+R\right)}^{3} \int u_{x}^{2} \psi_{x} \\
& \leq C e^{-\sqrt{\sigma}\left(c_{0}-\sigma^{\prime}\right)\left(t_{2}-t\right)} e^{-\sqrt{\sigma} x_{0}}+C \alpha_{0}^{\frac{3}{2}} \int u_{x}^{2} \psi_{x},
\end{aligned}
$$

and similarly,

$$
\int|u|^{5} \psi_{x} \leq C e^{-\sqrt{\sigma}\left(c_{0}-\sigma^{\prime}\right)\left(t_{2}-t\right)} e^{-\sqrt{\sigma} x_{0}}+C \alpha_{0}^{\frac{3}{2}} \int u^{2} \psi_{x}
$$

Estimate (A.1) follows, for $\alpha_{0}$ small enough (depending on $\sigma, \sigma^{\prime}, c_{0}, C_{0}$ ).

Integrating (A.1) on $\left[t_{1}, t_{2}\right]$, we get

$$
J_{x_{0}}\left(t_{2}\right)-J_{x_{0}}\left(t_{1}\right) \leq e^{-\sqrt{\sigma} x_{0}}
$$

We control the nonlinear term in $J_{x_{0}}(t)$ as before:

$$
\begin{aligned}
& \int|u|^{5} \psi \leq \int_{x<c_{0} t+R}|u|^{5} \psi+\int_{x>c_{0} t+R}|u|^{5} \psi \\
& \leq C \phi\left(\sqrt{\sigma}\left(R-\left(c_{0}-\sigma^{\prime}\right)\left(t_{2}-t\right)-x_{0}\right)\right)+\|u\|_{L^{\infty}\left(x>c_{0} t+R\right)}^{3} \int u^{2} \psi \\
& \leq C e^{-\sqrt{\sigma}\left(c_{0}-\sigma^{\prime}\right)\left(t_{2}-t\right)} e^{-\sqrt{\sigma} x_{0}}+C \alpha_{0}^{\frac{3}{2}} \int u^{2} \psi \leq C e^{-\sqrt{\sigma} x_{0}}+C \alpha_{0}^{\frac{3}{2}} \int u^{2} \psi .
\end{aligned}
$$

Thus, for $\alpha_{0}$ small enough,

$$
\begin{gathered}
J_{x_{0}}\left(t_{2}\right) \geq \int\left(u_{x}^{2}+u^{2}-C|u|^{5}\right)\left(t_{2}\right) \psi \\
\geq \int\left(u_{x}^{2}+u^{2}\right)\left(t_{2}\right) \psi-C e^{-\sqrt{\sigma} x_{0}}-C \alpha_{0}^{\frac{3}{2}} \int u^{2}\left(t_{2}\right) \psi \\
\geq \frac{3}{4} \int\left(u_{x}^{2}+u^{2}\right)\left(t_{2}, x\right) \phi\left(\sqrt{\sigma}\left(x-c_{0} t_{2}-x_{0}\right)\right) d x-C e^{-\sqrt{\sigma} x_{0}} ; \\
J_{x_{0}}\left(t_{1}\right) \leq \int\left(u_{x}^{2}+u^{2}+C|u|^{5}\right)\left(t_{1}\right) \psi \\
\leq \int\left(u_{x}^{2}+u^{2}\right)\left(t_{1}\right) \psi+C e^{-\sqrt{\sigma} x_{0}}+C \alpha_{0}^{\frac{3}{2}} \int u^{2}\left(t_{1}\right) \psi \\
\leq \frac{3}{2} \int\left(u_{x}^{2}+u^{2}\right)\left(t_{1}, x\right) \phi\left(\sqrt{\sigma}\left(x-c_{0} t_{1}-\left(c_{0}-\sigma^{\prime}\right)\left(t_{2}-t_{1}\right)-x_{0}\right)\right) d x+C e^{-\sqrt{\sigma} x_{0}} .
\end{gathered}
$$

Combining these estimates, we get

$$
\begin{aligned}
& \int\left(u_{x}^{2}+u^{2}\right)\left(t_{2}, x\right) \phi\left(\sqrt{\sigma}\left(x-c_{0} t-x_{0}\right)\right) d x \\
& \leq 2 \int\left(u_{x}^{2}+u^{2}\right)\left(t_{1}, x\right) \phi\left(\sqrt{\sigma}\left(x-c_{0} t_{1}-\left(c_{0}-\sigma^{\prime}\right)\left(t_{2}-t_{1}\right)-x_{0}\right)\right) d x+C e^{-\sqrt{\sigma} x_{0}} .
\end{aligned}
$$


A.2. Proof of Lemma 2.1. The proof of Lemma 2.1 is based on the three conservation laws, mass (1.2), energy (1.3) and integral (1.4).

Recall that

$$
\int Q_{c}^{2}=c^{\frac{1}{6}} \int Q^{2}, \quad E\left(Q_{c}\right)=c^{\frac{7}{6}} E(Q), \quad \int Q_{c}=\frac{1}{c^{\frac{1}{6}}} \int Q,
$$

Let $N \geq 2,0<c_{N}<\ldots<c_{1}$ and $\Delta_{1}, \Delta_{2}, \ldots, \Delta_{N} \in \mathbb{R}$. Let $u(t)$ be the solution of (1.1) satisfying (2.1). Let

$$
w(t)=u(t)-\sum_{j=1}^{N} Q_{c_{j}}\left(.-c_{j} t-\Delta_{j}\right) .
$$

By the uniqueness result in [11, we have, for some $C, \gamma>0$,

$$
\|w(t)\|_{H^{1}} \leq C e^{-\gamma t} .
$$

First, as in Step 1 of the proof of Lemma 1.4 using Lemma 3.1 and (A.2),

$$
\forall x_{0}>0, \forall t>0, \quad \int_{x<-x_{0}+c_{N} t} u^{2}(t, x) d x \leq C e^{-\sqrt{\frac{c_{N}}{2}} x_{0}} .
$$

Second, following the proof of Lemma 7.4 in [25], using the exponential decay in time (A.2), there exists $C>0$ such that

$$
\forall t>0, \quad \int_{x>c_{1} t}\left(x-c_{1} t\right)^{2} u^{2}(t, x) d x \leq C
$$

By the exponential decay properties of $Q_{c_{j}}$, we deduce from (A.3) and (A.4) that

$$
\forall t>0, \quad \int_{x<c_{N} t}\left(x-c_{N} t\right)^{2} w^{2}(t, x) d x+\int_{x>c_{1} t}\left(x-c_{1} t\right)^{2} w^{2}(t, x) d x \leq C .
$$

From this, we deduce easily that $\int|w(t)| \rightarrow 0$ as $t \rightarrow+\infty$. Indeed,

$$
\begin{aligned}
& \int|w(t)| \leq \int_{x<\frac{1}{2} c_{N} t}|w(t)|+\int_{\frac{1}{2} c_{N} t<x<\frac{3}{2} c_{1} t}|w(t)|+\int_{x>\frac{3}{2} c_{1} t}|w(t)| \\
& \leq C\left(\int_{x<\frac{1}{2} c_{N} t}\left(x-c_{N} t\right)^{\frac{3}{2}} w^{2}(t)\right)^{\frac{1}{2}}+C t^{\frac{1}{2}}\left(\int|w(t)|^{2}\right)^{\frac{1}{2}}+C\left(\int_{x>\frac{3}{2} c_{1} t}\left(x-c_{1} t\right)^{\frac{3}{2}} w^{2}(t)\right)^{\frac{1}{2}} \\
& \leq C t^{-\frac{1}{4}}+C e^{-\frac{\gamma}{4} t} .
\end{aligned}
$$

\section{Appendix B. Proof of Claim 4}

Set $D=\frac{B}{Q^{2}}, E=D Q^{\frac{3}{2}}=B Q^{-\frac{1}{2}}, F=E Q^{\frac{3}{2}}=D Q^{3}=B Q$. In particular,

$$
\int B Q^{\frac{5}{2}}=\int E Q^{3}=0 .
$$

First, we claim: 


\section{Claim 10.}

$$
\begin{aligned}
& \int L\left(B^{\prime}\right) B \frac{Q^{\prime}}{Q^{2}}=\int\left(D^{\prime}\right)^{2}\left(\frac{3}{2} Q^{3}+\frac{3}{10} Q^{6}\right)-\int D^{2}\left(3 Q^{3}-\frac{21}{5} Q^{6}+\frac{6}{5} Q^{9}\right), \\
& \int\left(D^{\prime}\right)^{2} Q^{3}=\int\left(E^{\prime}\right)^{2}+\frac{9}{4} \int E^{2}-\frac{9}{5} \int E^{2} Q^{3} \\
& \int\left(D^{\prime}\right)^{2} Q^{6}=\int\left(F^{\prime}\right)^{2}+9 \int F^{2}-\frac{27}{5} \int F^{2} Q^{3} .
\end{aligned}
$$

Proof of (B.1).

$$
\begin{aligned}
& \int L\left(B^{\prime}\right) B \frac{Q^{\prime}}{Q^{2}}=\int\left(D Q^{2}\right)^{\prime} L\left(D Q^{\prime}\right)=\int\left(2 D Q Q^{\prime}+D^{\prime} Q^{2}\right)\left(D L Q^{\prime}-2 D^{\prime} Q^{\prime \prime}-D^{\prime \prime} Q^{\prime}\right) \\
& =\int\left(D^{\prime}\right)^{2}\left(-2 Q^{2} Q^{\prime \prime}+\frac{1}{2}\left(Q^{2} Q^{\prime}\right)^{\prime}+2 Q\left(Q^{\prime}\right)^{2}\right)+\int D^{2}\left(2\left(Q Q^{\prime} Q^{\prime \prime}\right)^{\prime}-\left(Q\left(Q^{\prime}\right)^{2}\right)^{\prime \prime}\right) \\
& =\int\left(D^{\prime}\right)^{2}\left(-\frac{3}{2} Q^{2} Q^{\prime \prime}+3 Q\left(Q^{\prime}\right)^{2}\right)-\int D^{2}\left(\left(Q^{\prime}\right)^{3}\right)^{\prime} \\
& =\int\left(D^{\prime}\right)^{2}\left(\frac{3}{2} Q^{3}+\frac{3}{10} Q^{6}\right)-\int D^{2}\left(3 Q^{3}-\frac{21}{5} Q^{6}+\frac{6}{5} Q^{9}\right) .
\end{aligned}
$$

Proof of $(\underline{B .2})-($ B.3 $)$. Let $\beta>0$.

$$
\begin{aligned}
\int\left[\left(D Q^{1+\beta}\right)^{\prime}\right]^{2} & =\int\left(D^{\prime}\right)^{2} Q^{2(1+\beta)}+(1+\beta)^{2} \int D^{2}\left(Q^{\prime}\right)^{2} Q^{2 \beta}+2(1+\beta) \int D D^{\prime} Q^{\prime} Q^{1+2 \beta} \\
& =\int\left(D^{\prime}\right)^{2} Q^{2(1+\beta)}+D^{2}\left((1+\beta)^{2}\left(Q^{\prime}\right)^{2} Q^{2 \beta}-(1+\beta)\left(Q^{\prime} Q^{1+2 \beta}\right)^{\prime}\right) \\
& =\int\left(D^{\prime}\right)^{2} Q^{2(1+\beta)}+D^{2}\left(-\beta(1+\beta)\left(Q^{\prime}\right)^{2} Q^{2 \beta}-(1+\beta) Q^{\prime \prime} Q^{1+2 \beta}\right) \\
& =\int\left(D^{\prime}\right)^{2} Q^{2(1+\beta)}+(1+\beta) D^{2}\left(-(1+\beta) Q^{2+2 \beta}+\frac{2 \beta+5}{5} Q^{5+2 \beta}\right) .
\end{aligned}
$$

Thus, applied to $\beta=\frac{1}{2}$ and $\beta=2$,

$$
\begin{aligned}
& \int\left(D^{\prime}\right)^{2} Q^{3}=\int\left(E^{\prime}\right)^{2}+\frac{9}{4} \int E^{2}-\frac{9}{5} \int E^{2} Q^{3} \\
& \int\left(D^{\prime}\right)^{2} Q^{6}=\int\left(F^{\prime}\right)^{2}+9 \int F^{2}-\frac{27}{5} \int F^{2} Q^{3} .
\end{aligned}
$$

Note also that by definition of $E$ and $F$ :

$$
\int E^{2} Q^{3}=\int F^{2}
$$


End of the proof of Claim 4. We combine (B.1)-(B.2)-(B.3)-(B.5) to write $\int L\left(B^{\prime}\right) B \frac{Q^{\prime}}{Q^{2}}$ as a sum of two nonnegative quadratic forms in $E$ and $F$.

$$
\begin{aligned}
\int L\left(B^{\prime}\right) B \frac{Q^{\prime}}{Q^{2}} & =\frac{3}{2}\left(\int\left(E^{\prime}\right)^{2}+\frac{9}{4} \int E^{2}-\frac{9}{5} \int E^{2} Q^{3}\right) \\
& +\frac{3}{10}\left(\int\left(F^{\prime}\right)^{2}+9 \int F^{2}-\frac{27}{5} \int F^{2} Q^{3}\right) \\
& -3 \int E^{2}+\frac{21}{5} \int F^{2}-\frac{6}{5} \int F^{2} Q^{3} \\
& =\frac{3}{8} \int E^{2}+\frac{3}{2}\left(\int\left(E^{\prime}\right)^{2}-\frac{27}{5} \int E^{2} Q^{3}\right) \\
& +\frac{3}{10}\left(\int\left(F^{\prime}\right)^{2}+41 \int F^{2}-\frac{47}{5} \int F^{2} Q^{3}\right) .
\end{aligned}
$$

We claim

\section{Claim 11.}

$$
\begin{gathered}
\int\left(F^{\prime}\right)^{2}+\frac{35}{2} \int F^{2}-\frac{47}{5} \int F^{2} Q^{3} \geq 0 \\
\int\left(E^{\prime}\right)^{2}-\frac{27}{5} \int E^{2} Q^{3} \geq 0
\end{gathered}
$$

Proof. We use standard arguments from [31]. For $\beta>0$, the operator

$$
w^{\prime \prime}+\frac{1}{5} \beta(2 \beta+3) Q^{3} w
$$

has first eigenfunction $Q^{\beta}$ and first eigenvalue $-\beta^{2}$. In particular, for $\beta=4$, it follows that

$$
\forall w, \quad \int\left(w^{\prime}\right)^{2}+16 \int w^{2}-\frac{44}{5} \int Q^{3} w^{2} \geq 0
$$

Note that this can also be deduced from (B.4). Since $\frac{3}{5} Q^{3} \leq \frac{3}{2}$ from the expression of $Q$, we have proved (B.6).

We also know that for $\frac{3}{2}<\beta \leq 3$, the operator defined in (B.8) has exactly one other eigenfunction $Q^{\prime} Q^{\beta-\frac{5}{2}}$ with eigenvalue $-\left(\beta-\frac{3}{2}\right)^{2}$. In particular, with $\beta=3$,

$$
\int w Q^{3}=\int w Q^{\prime} Q^{\frac{1}{2}}=0 \Rightarrow \int\left(w^{\prime}\right)^{2}-\frac{27}{5} \int w^{2} Q^{3} \geq 0 .
$$

In conclusion,

$$
\int L\left(B^{\prime}\right) B \frac{Q^{\prime}}{Q^{2}} \geq \frac{3}{8} \int E^{2}+\frac{141}{10} \int E^{2} Q^{3} \geq \frac{3}{8} \int \frac{B^{2}}{Q}+\frac{141}{20} \int B^{2} Q^{2} .
$$

\section{Appendix C. Proof of Claim 6}

Here is an elementary claim that we will use frequently in this proof. The proof is immediate and we omit it. 
Claim 12. Let $F_{1}$ and $F_{2}$ be two $C^{\infty}$ functions such that for all $0 \leq k \leq 5$, $\sigma_{1}^{+}, \sigma_{1}^{-}, \sigma_{2}^{+}, \sigma_{2}^{-}>0, L>0$,

$$
\begin{gathered}
\left|F_{1}^{(k)}\right| \leq C_{k} e^{-\sigma_{1}^{+} x}, \text { for } x>0, \quad\left|F_{1}^{(k)}\right| \leq C_{k} e^{\sigma_{1}^{-} x}, \text { for } x<0 . \\
\left|F_{2}^{(k)}\right| \leq C_{k} e^{-\sigma_{2}^{+}(x+L)}, \text { for } x>-L, \quad\left|F_{2}^{(k)}\right| \leq C_{k} e^{\sigma_{2}^{-}(x+L)}, \text { for } x<-L .
\end{gathered}
$$

Then, for all $k \geq 0$,

$$
\begin{array}{ll}
\text { if } \sigma_{1}^{-} \neq \sigma_{2}^{+}: & \left\|F_{1} F_{2}\right\|_{H^{4}} \leq C_{k}^{\prime} e^{-\min \left(\sigma_{1}^{-}, \sigma_{2}^{+}\right) L}, \\
\text { if } \sigma_{1}^{-}=\sigma_{2}^{+}: & \left\|F_{1} F_{2}\right\|_{H^{4}} \leq C_{k}^{\prime} L e^{-\sigma_{1}^{-} L},
\end{array}
$$

Since for all $p \geq 0,\left|R_{j}^{(p)}\right| \lesssim e^{-\sqrt{c_{j}}\left(x-c_{j} t\right)}$ by Claim 12, we easily check that for $j_{k} \neq j_{l}$,

$$
\left\|R_{j_{1}}^{2} R_{j_{2}}^{2}\right\|_{H^{4}} \lesssim e^{-2 \sigma_{0} t}, \quad\left\|R_{j_{1}}^{2} R_{j_{2}} R_{j_{3}}\right\|_{H^{4}} \lesssim e^{-3 \sigma_{0} t}, \quad\left\|R_{j_{1}} R_{j_{2}} R_{j_{3}} R_{j_{4}}\right\|_{H^{4}} \lesssim e^{-4 \sigma_{0} t}
$$

and thus

$$
\left\|E_{1}\right\|_{H^{4}} \lesssim e^{-2 \sigma_{0} t}
$$

Note that for $k \geq 0$,

$$
\begin{aligned}
\forall x, & \left|Q^{(k)}(x)-(-\operatorname{sign} x)^{k}(10)^{\frac{1}{3}} e^{-|x|}\right| \leq C_{k} e^{-4|x|}, \\
& \left|Q_{c_{j}}^{(k)}(x)-(-\operatorname{sign} x)^{k}(10)^{\frac{1}{3}} c_{j}^{\frac{k}{2}+\frac{1}{3}} e^{-\sqrt{c_{j}}|x|}\right| \leq C_{k} e^{-4 \sqrt{c_{j}}|x|} .
\end{aligned}
$$

To estimate $E_{2}$, we consider two different regions in space. Assume $k>j$. For $x>y_{k}:=c_{k} t+\Delta_{k}$, we have

$$
\begin{aligned}
& \left\|\left(4 R_{k}(t, x)-z_{j, k}(t) e^{-\sqrt{c_{k}}\left(x-y_{j}\right)}\right) R_{c_{j}}^{3}\right\|_{H^{3}\left(x>y_{k}\right)} \\
& =4\left\|\left(Q_{c_{k}}\left(x-y_{k}\right)-(10)^{\frac{1}{3}} c_{k}^{\frac{1}{3}} e^{-\sqrt{c_{k}}\left(\Delta_{j}-\Delta_{k}\right)} e^{-\sqrt{c_{k}}\left(c_{j}-c_{k}\right) t} e^{-\sqrt{c_{k}}\left(x-y_{j}\right)}\right) R_{j}^{3}\right\|_{H^{3}\left(x>y_{k}\right)} \\
& =4\left\|\left(Q_{c_{k}}\left(x-y_{k}\right)-(10)^{\frac{1}{3}} c_{k}^{\frac{1}{3}} e^{-\sqrt{c_{k}}\left(x-y_{k}\right)}\right) R_{j}^{3}\right\|_{H^{3}\left(x>y_{k}\right)} \lesssim e^{-2 \sigma_{0} t} . \\
& \leq C\left\|e^{-4 \sqrt{c_{k}}\left(x-y_{k}\right)} e^{-3 \sqrt{c_{j}}\left|x-y_{j}\right|}\right\|_{L^{2}\left(x>y_{k}\right)} \lesssim
\end{aligned}
$$

For $x<y_{k}$, the following is straightforward

$$
\left\|z_{j} e^{-\sqrt{c_{k}}\left(x-y_{k}\right)} R_{j}^{3}\right\|_{H^{3}\left(x<y_{k}\right)}+\left\|R_{k} R_{j}^{3}\right\|_{H^{3}\left(x<y_{k}\right)} \lesssim e^{-2 \sigma_{0} t} .
$$

The case $k<j$ is similar. We obtain

$$
\left\|E_{2}\right\|_{H^{4}} \lesssim e^{-2 \sigma_{0} t}
$$

By the definition of $z_{j, k}$, it is clear that quadratic and higher order terms in $Z$ in the expression of $E_{3}$ are controlled by $e^{-2 \sigma_{0} t}$, i.e.

$$
\left\|Z^{4}\right\|_{H^{4}}+\left\|R Z^{3}\right\|_{H^{4}}+\left\|R^{2} Z^{2}\right\|_{H^{4}} \leq e^{-2 \sigma_{0} t} .
$$

We now consider the remaining term in $E_{3}$, which we can write as follows

$$
\tilde{E}_{3}=4 \sum_{\substack{j \neq k \\ l_{1}, l_{2}, l_{3} \notin L_{j}}} R_{l_{1}} R_{l_{2}} R_{l_{3}} Z_{j, k},
$$


where $L_{j}=\left\{\left(l_{1}, l_{2}, l_{3}\right) \mid l_{1}=l_{2}=l_{3}=j\right.$ or $\left.l_{1}=l_{2}=l_{3}<j\right\}$. In the sum defining $\tilde{E}_{3}$, if $l_{1} \neq l_{2}$, then

$$
\left\|R_{l_{1}} R_{l_{2}}\right\|_{H^{4}} \lesssim e^{-\sigma_{0} t}
$$

and thus, the decay of $Z_{j, k}$,

$$
\left\|R_{l_{1}} R_{l_{2}} R_{l_{3}} Z_{j, k}\right\|_{H^{4}} \lesssim e^{-2 \sigma_{0} t} .
$$

Therefore, we only have to consider terms such that $l_{1}=l_{2}=l_{3}=l>j$. For such $j, k, l$, we have immediately, by the decay of $R_{l}$,

$$
\left\|R_{l}^{3} Z_{j, k}\right\|_{H^{4}\left(x>y_{j}\right)} \lesssim e^{-2 \sigma_{0} t} .
$$

For $y<y_{j}$, we use the space decay of $A_{j, k}$ on the left given in (4.49) and (4.54),

$$
\left\|R_{l}^{3} Z_{j, k}\right\|_{H^{4}\left(x<y_{j}\right)} \lesssim e^{-\sigma_{0} t}\left\|e^{-3 \sqrt{c_{l}}|x|} e^{-\gamma_{j, k}^{0}\left|x-\left(c_{j}-c_{l}\right) t\right|}\right\|_{L^{2}\left(x<y_{j}-y_{l}\right)} \lesssim e^{-2 \sigma_{0} t} .
$$

We have just proved

$$
\left\|E_{3}\right\|_{H^{4}} \lesssim e^{-2 \sigma_{0} t}
$$

For $E_{4}$, we use (4.49), (4.55) and we argue as before for $E_{2}$.

$$
E_{4}=\sum_{j \neq k, j>l}\left(4 Z_{j, k}-z_{j, k}^{\mathrm{I}} e^{-\gamma_{j, k}^{\mathrm{I}}\left(x-y_{l}\right)}-z_{j, k}^{\mathrm{II}} e^{-\gamma_{j, k}^{\mathrm{II}}\left(x-y_{l}\right)}\right) R_{l}^{3} .
$$

For $j \neq k, j>l$,

$$
\begin{aligned}
\left\|\left(Z_{j, k}-z_{j, k}^{\mathrm{I}} e^{-\gamma_{j, k}^{\mathrm{I}}\left(x-y_{l}\right)}-z_{j, k}^{\mathrm{II}} e^{-\gamma_{j, k}^{\mathrm{II}}\left(x-y_{l}\right)}\right) R_{l}^{3}\right\|_{H^{4}\left(x>y_{j}\right)} & \lesssim\left|z_{j, k}\right|\left\|e^{-\frac{9}{8}\left(x-y_{j}\right)} e^{-3 \sqrt{c_{l}}\left|x-y_{l}\right|}\right\|_{L^{2}\left(x>y_{j}\right)} \\
& \lesssim e^{-2 \sigma_{0} t} .
\end{aligned}
$$

The estimate for $y<y_{j}$ is immediate and we obtain

$$
\left\|E_{4}\right\|_{H^{4}} \lesssim e^{-2 \sigma_{0} t}
$$

Finally, we consider $E_{5}$. As for $E_{3}$, it is clear that quadratic and higher order terms in $W$ in the expression of $E_{5}$ are controlled by $e^{-2 \sigma_{0} t}$, i.e.

$$
\left\|(R+Z)^{2} W^{2}\right\|_{H^{4}}+\left\|(R+Z) W^{3}\right\|_{H^{4}}+\left\|W^{4}\right\|_{H^{4}} \lesssim e^{-2 \sigma_{0} t} .
$$

Similary, terms containing products of $Z_{j, k}$ and $Z_{j, k, l}^{\mathrm{I}, \mathrm{II}}$ are also controlled directly by the expression of $Z_{j, k}$ :

$$
\left\|\left((R+Z)^{3}-R^{3}\right) W\right\|_{H^{4}} \leq e^{-2 \sigma_{0} t} .
$$

Therefore, it only remains to estimate the following term

$$
\begin{aligned}
& R^{3} W-\sum_{j=2}^{N} \sum_{\substack{k=1 \\
k \neq j}}^{N} \sum_{l=1}^{j-1} R_{l}^{3}\left(Z_{j, k, l}^{\mathrm{I}}+Z_{j, k, l}^{\mathrm{II}}\right) \\
& =\sum_{l_{1}, l_{2}, l_{3}} R_{l_{1}} R_{l_{2}} R_{l_{3}} W-\sum_{j=2}^{N} \sum_{\substack{k=1 \\
k \neq j}}^{N} \sum_{l=1}^{j-1} R_{l}^{3}\left(Z_{j, k, l}^{\mathrm{I}}+Z_{j, k, l}^{\mathrm{II}}\right) .
\end{aligned}
$$


In the first sum on the right-hand side term, when $l_{1} \neq l_{2}$ or $l_{1} \neq l_{3}$ or $l_{2} \neq l_{3}$, the corresponding term is immediately controlled:

$$
\left\|\sum_{\substack{l_{1}, l_{2}, l_{3} \\ l_{n} \neq l_{m}}} R_{l_{1}} R_{l_{2}} R_{l_{3}} W\right\|_{H^{4}} \lesssim e^{-2 \sigma_{0} t} .
$$

Thus, it only remains to consider terms:

$$
\sum_{l_{1}=1}^{N} \sum_{j=2}^{N} \sum_{\substack{k=1 \\ k \neq j}}^{N} \sum_{\substack{l_{2}=1 \\ l_{2} \neq l_{1}}}^{j-1} R_{l_{1}}^{3}\left(Z_{j, k, l_{2}}^{\mathrm{I}}+Z_{j, k, l_{2}}^{\mathrm{II}}\right) .
$$

To estimate each term of this sum, we distinguish the cases $l_{1}>l_{2}$ and $l_{2}>l_{1}$. For $l_{1}>l_{2}$, we use the estimate of $A_{j, k, l_{2}}^{\mathrm{I}, \mathrm{II}}$ for $x<0$ in (4.59). Indeed,

$$
\left\|R_{l_{1}}^{3} Z_{j, k, l_{2}}^{\mathrm{I}, \mathrm{II}}\right\|_{H^{4}\left(x<y_{l_{2}}\right)} \lesssim\left|z_{j, k, l_{2}}^{\mathrm{I}, \mathrm{II}}\right|\left\|e^{-3 \sqrt{c_{l_{1}}}|x|} e^{-\sqrt{c_{l_{2}}}\left|x-\left(c_{l_{2}}-c_{l_{1}}\right) t\right|}\right\|_{L^{2}\left(x<y_{l_{2}}-y_{l_{1}}\right)} \lesssim e^{-2 \sigma_{0} t} .
$$

and a similar estimate for $x>y_{l_{2}}$ is clear.

For the case $l_{1}<l_{2}$, we argue similarly, but we use the estimate in (4.59) for $x>0$ and the exact expression of $z_{j, k, l_{2}}^{\mathrm{I}, \mathrm{II}}$,

$$
\begin{aligned}
\left\|R_{l_{1}}^{3} Z_{j, k, l_{2}}^{\mathrm{I}, \mathrm{II}}\right\|_{H^{4}\left(x>y_{l_{2}}\right)} & \lesssim\left|z_{j, k, l_{2}}^{\mathrm{I}, \mathrm{II}}\right| \mid e^{-3 \sqrt{c_{l_{1}}}|x|} e^{-\left(\sqrt{c_{l_{2}}}-\gamma_{j, k}^{\mathrm{I}, \mathrm{II}}\right)\left(x-\left(c_{l_{1}}-c_{l_{2}}\right) t\right)} \|_{L^{2}\left(x>y_{l_{1}}-y_{l_{2}}\right)} \\
& \lesssim e^{-\sqrt{c_{k}}\left|c_{j}-c_{k}\right| t} e^{-\gamma_{j, k}^{\mathrm{I}, \mathrm{II}}\left(c_{l_{2}}-c_{j}\right) t} e^{-\left(\sqrt{c_{l_{2}}}-\gamma_{j, k}^{\mathrm{I}, \mathrm{II}}\right)\left(c_{l_{1}}-c_{l_{2}}\right) t} .
\end{aligned}
$$

Let $j_{1}$ be such that $c_{j_{1}}-c_{j_{1}+1}=\min _{j}\left(c_{j}-c_{j+1}\right)$. We have

$$
\begin{gathered}
\left(\sqrt{c_{l_{2}}}-\gamma_{j, k}^{\mathrm{I}, \mathrm{II}}\right)\left(c_{l_{1}}-c_{l_{2}}\right) \geq\left(\sqrt{c_{l_{2}}}-\gamma_{j, k}^{\mathrm{I}, \mathrm{II}}\right)\left(c_{j_{1}}-c_{j_{1}+1}\right), \\
\gamma_{j, k}^{\mathrm{I}, \mathrm{II}}\left(c_{l_{2}}-c_{j}\right) \geq \gamma_{j, k}^{\mathrm{I}, \mathrm{II}}\left(c_{j_{1}}-c_{j_{1}+1}\right) .
\end{gathered}
$$

Thus

$$
\left\|R_{l_{1}}^{3} Z_{j, k, l_{2}}^{\mathrm{I}, \mathrm{II}}\right\|_{H^{4}\left(x>y_{l_{2}}\right)} \lesssim e^{-\sqrt{c_{k}}\left|c_{j}-c_{k}\right| t} e^{-\sqrt{c_{l_{2}}}\left(c_{j_{1}}-c_{j_{1}+1}\right) t} \lesssim e^{-2 \sigma_{0} t}
$$

The estimate for $x<y_{l_{2}}$ is clear for this term.

Thus, we have proved

$$
\left\|E_{5}\right\|_{H^{4}} \lesssim e^{-2 \sigma_{0} t}
$$

\section{References}

[1] W. Craig, P. Guyenne, J. Hammack, D. Henderson and C. Sulem, Solitary water wave interactions. Phys. Fluids 18, (2006), 57-106.

[2] E. Fermi, J. Pasta and S. Ulam, Studies of nonlinear problems, I, Los Alamos Report LA1940 (1955); reproduced in Nonlinear Wave Motion, A.C. Newell, ed., American Mathematical Society, Providence, R. I., 1974, pp. 143-156.

[3] J. Hammack, D. Henderson, P. Guyenne and Ming Yi, Solitary-wave collisions, in Proceedings of the 23rd ASME Offshore Mechanics and Artic Engineering (A symposium to honor Theodore Yao-Tsu Wu), Vancouver, Canada, June 2004 (Word Scientific, Singapore, 2004).

[4] R. Hirota, Exact solution of the Korteweg-de Vries equation for multiple collisions of solitons, Phys. Rev. Lett., 27 (1971), 1192-1194.

[5] T. Kato, On the Cauchy problem for the (generalized) Korteweg-de Vries equation. Studies in applied mathematics, 93-128, Adv. Math. Suppl. Stud., 8, Academic Press, New York, 1983. 
[6] C.E. Kenig, G. Ponce and L. Vega, Well-posedness and scattering results for the generalized Korteweg-de Vries equation via the contraction principle, Comm. Pure Appl. Math. 46, (1993) $527-620$.

[7] H. Koch and J. Marzuolas, Small data scattering and soliton stability in $H^{-1 / 6}$ for the quartic KdV equation, Analysis \& PDE 5 (2012), 145-198.

[8] M. D. Kruskal, The Korteweg-de Vries equation and related evolution equations, in Nonlinear Wave Motion, A.C. Newell, ed., American Mathematical Society, Providence, R. I., 1974, pp. 61-83.

[9] P. D. Lax, Integrals of nonlinear equations of evolution and solitary waves, Comm. Pure Appl. Math. 21, (1968) 467-490.

[10] J. H. Maddocks and R. L Sachs, On the stability of KdV multi-solitons, Comm. Pure Appl. Math. 46 (1993), 867-901.

[11] Y. Martel, Asymptotic $N$-soliton-like solutions of the subcritical and critical generalized Korteweg-de Vries equations, Amer. J. Math. 127 (2005), 1103-1140.

[12] Y. Martel, Linear problems related to asymptotic stability of solitons of the generalized KdV equations, SIAM J. Math. Anal. 38 (2006), 759-781.

[13] Y. Martel and F. Merle, Asymptotic stability of solitons for subcritical generalized KdV equations, Arch. Ration. Mech. Anal. 157, (2001) 219-254.

[14] Y. Martel and F. Merle, Asymptotic stability of solitons for subcritical (gKdV) equations revised, Nonlinearity 18, (2005) 55-80.

[15] Y. Martel and F. Merle, Stability of blow-up profile and lower bounds for blow-up rate for the critical generalized KdV equation. Ann. Math. 155 (2002), 235-280.

[16] Y. Martel and F. Merle, Asymptotic stability of solitons of the (gKdV) equations with a general nonlinearity. Math. Ann. 341 (2008), 391-427.

[17] Y. Martel and F. Merle, Stability of two soliton collision for nonintegrable (gKdV) equations, Comm. Math. Phys. 286 (2009), 39-79.

[18] Y. Martel and F. Merle, Description of two soliton collision for the quartic (gKdV) equation, Ann. Math. 174 (2011), 757-857.

[19] Y. Martel and F. Merle, Inelastic interaction of nearly equal solitons for the quartic (gKdV) equation, Invent. Math. 183 (2011), 563-648,

[20] Y. Martel, F. Merle and T. Mizumachi, Description of the inelastic collision of two solitary waves for the BBM equation, Arch. Ration. Mech. Anal. 196 (2010), 517-574.

[21] Y. Martel, F. Merle and Tai-Peng Tsai, Stability and asymptotic stability in the energy space of the sum of $N$ solitons for the subcritical (gKdV) equations, Commun. Math. Phys. 231, (2002) 347-373.

[22] R.M. Miura, The Korteweg-de Vries equation: a survey of results, SIAM Review 18, (1976) 412-459.

[23] T. Mizumachi, Weak interaction between solitary waves of the generalized KdV equations, SIAM J. Math. Anal. 35 (2003), 1042-1080.

[24] C. Munoz, On the inelastic 2-soliton collision for generalized KdV equations, Int. Math. Res. Notices (2010), 1624-1719.

[25] C. Munoz, Soliton dynamics for generalized ( $\mathrm{gKdV}$ ) equations in a slowly varying medium, Analysis and PDE (2011), 573-638.

[26] C. Munoz, On the soliton dynamics under slowly varying medium for NLS equations, Math. Annalen (2012), 867-943.

[27] R. L. Pego and M. I. Weinstein, Asymptotic stability of solitary waves, Commun. Math. Phys., 164 (1994) 305-349.

[28] G. Perelman, Two soliton collision for nonlinear Schrödinger equations in dimension 1. Ann. Inst. H. Poincaré Anal. Non Linéaire 28 (2011), 357-384.

[29] L.Y. Shih, Soliton-like interaction governed by the generalized Korteweg-de Vries equation, Wave motion 2 (1980), 197-206.

[30] T. Tao, Scattering for the quartic generalised Korteweg-de Vries equation, J. Diff. Eqs. 232 (2007), 623-651.

[31] E.C. Titchmarsh, Eigenfunction Expansions Associated with Second-order Differential Equations, Oxford, Clarendon Press, 1946. 
[32] M. Wadati and M. Toda, The exact $N$-soliton solution of the Korteweg-de Vries equation, J. Phys. Soc. Japan 32, (1972) 1403-1411.

[33] P.D. Weidman and T. Maxworthy, Experiments on strong interactions between solitary waves, J. Fluids Mech. 85, (1978) 417-431.

[34] M. I. Weinstein, Lyapunov stability of ground states of nonlinear dispersive evolution equations, Comm. Pure Appl. Math. 39, (1986) 51-68.

[35] N.J. Zabusky, Solitons and energy transport in nonlinear lattices, Computer Physics Communications, 5 (1973), 1-10.

[36] N.J. Zabusky and M.D. Kruskal, Interaction of "solitons" in a collisionless plasma and recurrence of initial states, Phys. Rev. Lett. 15 (1965), 240-243.

Ecole Polytechnique, CMLS CNRS UMR7640

E-mail address: yvan.martel@polytechnique.edu

Université de Cergy Pontoise and Institut des Hautes Études Scientifiques, AGM CNRS UMR8088

E-mail address: merle@math.u-cergy.fr 